\title{
Equilibrium Time-Consistent Strategy for Corporate International Investment Problem with Mean-Variance Criterion
}

\author{
Jun Long ${ }^{1,2}$ and Sanyun Zeng ${ }^{3}$ \\ ${ }^{1}$ School of Mathematics, Sun Yat-sen University, Guangzhou 510275, China \\ ${ }^{2}$ School of Preparatory Education for Minority Nationalities, Jishou University, Hunan 416000, China \\ ${ }^{3}$ School of Accounting, Guangdong University of Foreign Studies, Guangzhou 510006, China
}

Correspondence should be addressed to Sanyun Zeng; zengsanyun@163.com

Received 12 May 2016; Revised 17 July 2016; Accepted 25 August 2016

Academic Editor: Vyacheslav Kalashnikov

Copyright (C) 2016 J. Long and S. Zeng. This is an open access article distributed under the Creative Commons Attribution License, which permits unrestricted use, distribution, and reproduction in any medium, provided the original work is properly cited.

We analyze a continuous-time model for corporate international investment problem (CIIP) with mean-variance criterion. Based on Nash subgame perfect equilibrium theory, we define an infinitesimal operator and directly derive an extended Hamilton-JacobiBellman (HJB) equation. Besides, we also obtain the equilibrium time-consistent strategy for CIIP. In addition, we discuss two cases of risk aversion coefficient; one is constant and the other is state dependent. Finally, the simulation results are given to illustrate our conclusions and the influence of some parameters on the optimal solution.

\section{Introduction}

Generally, a continuous-time stochastic optimal control problem will lead to time inconsistency if the functional has the following form:

$$
\begin{aligned}
& J(t, w, \pi) \\
& =E_{t, w}\left[\int_{t}^{T} C\left(w, W_{s}^{\pi} ; \pi\left(W_{s}^{\pi}\right)\right) d s+F\left(w, W_{T}^{\pi}\right)\right] \\
& \quad+G\left(w, E_{t, w}\left[W_{T}^{\pi}\right]\right),
\end{aligned}
$$

where $W_{s}$ is a controlled Markov process and $W_{t}=w$. The term $\pi$ is a control applied at time $s$. The local utility function $C$, the terminal utility function $F$, and the nonlinear function $G$ are given functions. The reasons are as follows:

(1) For a fixed initial point $\left(t, W_{t}\right)$, we can determine the optimal control law $\hat{\pi}$ through maximizing the functional $J\left(t, W_{t}, \pi\right)$. But the control law $\hat{\pi}$ at a later point $\left(s, W_{s}\right)$ will no longer be optimal for the functional $J\left(s, W_{s}, \pi\right)$. This will lead to a timeinconsistent problem.
(2) If the term $E_{t, w}\left[F\left(w, W_{T}^{\pi}\right)\right]$ depends on the wealth $W_{t}=w$ at time $t$, this will also lead to a timeinconsistent problem.

(3) Generally, the term $G\left(w, E_{t, w}\left[W_{T}^{\pi}\right]\right)$ is not an expected value of a nonlinear function of terminal wealth but a nonlinear function of the expected value of terminal wealth, and this will also lead to a time-inconsistent problem.

When $C \equiv 0$, (1) becomes a mean-variance criterion problem. In this paper, we study corporate international investment problem with mean-variance criterion which is time inconsistent.

Because time-inconsistent problem does not satisfy Bellman's optimality principle, the standard dynamic programming approach fails. In order to deal with time inconsistency, there are three common ways to handle this problem:

(1) We dismiss the entire problem as being silly.

(2) We fix one initial point, for example, $\left(0, W_{0}\right)$, and then try to find the control law $\hat{\pi}$ which maximizes $J\left(0, W_{0}, \pi\right)$. We simply consider that, at a later time 
point such as $\left(s, W_{s}\right)$, the control law $\hat{\pi}$ is still optimal for the functional $J\left(s, W_{s}, \pi\right)$. This is well known as precommitment in the economics literature.

(3) Using game theory approach, we can seriously solve the time-inconsistent problem.

The game theory approach which used Nash equilibrium points to handle time inconsistency has a long history which started with Strotz [1], in which a deterministic Ramsay problem was studied. Further works in continuous and discrete time along this line are provided in Goldman [2], Krusell and Smith [3], Peleg and Yaari [4], Pollak [5], Vieille and Weibull [6], Björk and Murgoci [7], and so on.

More specifically, Björk and Murgoci [7] deduced an extended HJB equation in a discrete time setting and extended it to continuous-time setting by taking formal limits for the discrete problem. Hu et al. [8] studied a general time-inconsistent stochastic linear-quadratic (LQ) control problem and derived a sufficient condition for equilibrium control via a flow of forward-backward stochastic differential equations. Yong [9] studied a time-inconsistent optimal control problem and proved the existence of an equilibrium control via a forward ordinary differential equation coupled with a backward Riccati-Volterra integral equation. Yong [10] considered a general time-inconsistent optimal control problem for stochastic differential equations with deterministic coefficients. Under suitable conditions, they derived a Hamilton-Jacobi-Bellman equation for the equilibrium value function of the problem. Czichowsky [11] developed a timeconsistent formulation of mean-variance portfolio problem and proved the convergence based on a global description of the locally optimal strategy in terms of the structure condition and the Föllmer-Schweizer decomposition of the meanvariance tradeoff. Yong [12] considered a linear-quadratic optimal control problem for mean-field stochastic differential equations with deterministic coefficients and presented open-loop and closed-loop equilibrium solutions for such kind of problems. Björk and Murgoci [13] studied the problem with state-dependent risk aversion in the sense that the risk aversion is inversely proportional to the current wealth, and they showed that the time-consistent control was linear in wealth. Bensoussan et al. [14] studied the time-consistent strategies in the mean-variance portfolio selection with shortselling prohibition in both discrete and continuous-time settings. Using backward induction, the equilibrium control was shown to be linear in wealth. Wang and $\mathrm{Wu}$ [15] studied a partially observed recursive optimization problem. They established Kalman-Bucy filtering equations for a family of parameterized forward and backward stochastic differential equations and obtained the equilibrium control by means of backward separation technique. Cui et al. [16] investigated the effects of portfolio constraints on time consistency of efficiency for convex cone constrained markets and derived the semianalytical expressions for the precommitted efficient mean-variance policy and the minimum-variance signed supermartingale measure (VSSM) and revealed their close relationship. Cui et al. [17] considered a continuous-time mean-variance portfolio optimization problem and derived an explicit time-consistent investment policy.
In addition, there are many literatures concerning time consistency in other areas, for example, reinsurance (Zeng and $\mathrm{Li}$ [18], Li et al. [19], Zeng et al. [20], Y. Li and Z. Li [21], Zhao et al. [22], Zhao et al. [23], Li et al. [24], Li et al. [25], Liang and Song [26], and Li et al. [27]), asset-liability management (Wei et al. [28]), pricing (Pirvu and Zhang [29]), dividend strategies (Chen et al. [30]), and jumps (Zeng et al. [20] and Dang and Forsyth [31]).

In recent years, there are some literatures on corporate international investment problem (CIIP). To be more specific, Choi [32] considered the CIIP in which the quantity of output is assumed to be unity, and the dynamic processes of price are assumed to follow Bachelier model. Based on Choi [32], Bellalah and Wu [33] assumed that the quantity of output is a function of output price and the dynamic processes follow geometric Brownian motion model. Their model accounted for the effects of exchange rates, taxes, and information costs on corporate international investment and assumed that firm maximized the present value of manager's expected utility of net cash flows. Finally, they made a conclusion that information played a central role in foreign investments and explained the "home bias" in international finance. Besides, Wu and Zhang [34], D. Zhang and T. Zhang [35], and Huang and Zhang [36] also studied corporate international investment problem.

However, these prior literatures did not take into account time inconsistency. So, our study tries to explore this problem. In other words, we study the time-inconsistent problem of corporate international investment and deduce a timeconsistent strategy for it.

This study contributes to the literature as follows. In the first place, based on $\mathrm{Wu}$ and Zhang [34] which considered firms only invested in a real project (home market) and money account and Bellalah and $\mathrm{Wu}$ [33] which considered firms only invested in home and foreign market, we investigate those who invest not only in home and foreign market, but also in money account. There is one more point; based on Nash subgame perfect equilibrium theory, we define an infinitesimal operator and directly derive an extended HJB system for the general optimization problem from the prospective of continuous time. Last but not least, combining Björk and Murgoci [7] with Bellalah and Wu [33], we obtain equilibrium time-consistent strategy for corporate international investment problem. In addition, inspired by Björk et al. [37] and Y. Li and Z. Li [21], we discuss not only constant risk aversion coefficient but also state-dependent risk aversion coefficient.

This paper is organized as follows. In Section 2, we introduce the general optimization problem with meanvariance criterion in continuous time and derive an extended HJB equation directly. In Section 3, we apply this method to corporate international investment problem (CIIP) and deduce the equilibrium time-consistent strategy for CIIP. Some examples illustrating the main features of model are explicitly solved in Section 4. Section 5 contains the main conclusions and some extensions. In the end, some technical details are provided in Appendix. 


\section{The General Optimization Problem with Mean-Variance in Continuous-Time Setting}

Björk and Murgoci [7] discussed the time consistency in a discrete time setting and extended it to a continuous-time setting via limit theory, but their process is complicated. In this section, we focus on the continuous-time problem directly. Based on Nash subgame perfect equilibrium theory, we, applying the dynamic programming approach, define an infinitesimal operator and derive an extended HJB system which is consistent with Björk and Murgoci [7] in continuous time. Compared to Björk and Murgoci [7], our approach avoids the complicated transformation process from discrete time to continuous time. Therefore, our process is more simple and straightforward. To some extent, this study is a good supplement to Björk and Murgoci [7] in continuous time.

In this section, we consider a controlled Markov process in continuous time on the time interval $[0, T]$. Define the measurable state space $\mathscr{W}$ and the measurable control space $\Pi$. The dynamic state process is given by

$$
d W_{t}=\mu\left(t, W_{t}, \pi_{t}\right) d t+\sigma\left(t, W_{t}, \pi_{t}\right) d B_{t},
$$

where $W_{t} \in \mathscr{W}$ and $\pi_{t} \in \Pi$.

2.1. Basic Problem Formulation. For initial point $(t, w) \in$ $[0, T] \times \mathscr{W}$, with a fixed control law $\pi$, we consider the simplified functional

$$
J(t, w, \pi)=E_{t, w}\left[F\left(w, W_{T}^{\pi}\right)\right]+G\left(w, E_{t, w}\left[W_{T}^{\pi}\right]\right) .
$$

Firstly, we introduce some definitions as follows.

Definition 1. For each $\pi \in \Pi$ and efficient small number $\varepsilon>0$, an operator $P^{\pi}$, operating on real valued functions of the form $h(t, w)$, is defined by

$$
P^{\pi} h(t, w)=E\left[h\left(t+\varepsilon, W_{t+\varepsilon}^{\pi}\right) \mid W_{t}=w\right] .
$$

A corresponding infinitesimal operator $A^{\pi}$ is defined by

$$
A^{\pi}=P^{\pi}-I
$$

where $I$ is the identity operator.

Therefore, the infinitesimal operator $A^{\pi}$ can be expressed as

$$
A^{\pi} h(t, w)=E_{t, w}\left[h\left(t+\varepsilon, W_{t+\varepsilon}^{\pi}\right)\right]-h(t, w) .
$$

The way that controls are influencing the dynamics of process is formalized by specifying the controlled infinitesimal generator of $W_{t}$.

Definition 2. Consider a control law $\widehat{\pi}$ (informally viewed as a candidate equilibrium law). For any efficient small number $\varepsilon>0$, one chooses a fixed $\pi \in \Pi$ and also fixes an arbitrarily chosen initial point $(t, w)$. The control law $\pi_{\varepsilon}$ is defined by

$$
\pi_{\varepsilon}(s, w)= \begin{cases}\pi, & \text { if } s \in[t, t+\varepsilon), \\ \widehat{\pi}, & \text { if } s \in[t+\varepsilon, T] .\end{cases}
$$

If

$$
\liminf _{\varepsilon \rightarrow 0} \frac{J\left(t, W_{t}, \widehat{\pi}\right)-J\left(t, W_{t}, \pi_{\varepsilon}\right)}{\varepsilon} \geq 0,
$$

for all $\pi \in \Pi$, one says that $\hat{\pi}$ is an equilibrium control law. The equilibrium value function $V$ is defined by

$$
V\left(t, W_{t}\right)=J\left(t, W_{t}, \widehat{\pi}\right) .
$$

2.2. Deriving the Extended HJB System. For convenient description, we use the symbol $\left(t, w ; W_{T}^{\pi}\right)$ or $\left(t, w, y ; W_{T}^{\pi}\right)$ to replace $(t, w)$ or $(t, w, y)$, although $W_{T}^{\pi}$ may be a determined scalar but not a variable.

Denote that

$$
\begin{aligned}
f\left(t, w, y ; W_{T}^{\pi}\right) & =E_{t, w}\left[F\left(y, W_{T}^{\pi}\right)\right], \\
g\left(t, w ; W_{T}^{\pi}\right) & =E_{t, w}\left[W_{T}^{\pi}\right], \\
f^{y}\left(t, w ; W_{T}^{\pi}\right) & =f\left(t, w, y ; W_{T}^{\pi}\right), \quad \text { if } y \text { is fixed, } \\
G^{w}\left(g\left(t, y ; W_{T}^{\pi}\right)\right) & =G\left(w, g\left(t, y ; W_{T}^{\pi}\right)\right),
\end{aligned}
$$

if $w$ is fixed,

$$
\begin{aligned}
(G \diamond g)\left(t, w ; E_{T}^{\pi}\right)= & G\left(w, g\left(t, w ; E_{T}^{\pi}\right)\right), \\
\left(H_{g}^{\pi} G\right)\left(t, w ; E_{T}^{\pi}\right)= & G^{w}\left(P^{\pi}\left(g\left(t, w ; W_{T}^{\pi}\right)\right)\right) \\
& -G^{w}\left(g\left(t, w ; W_{T}^{\pi}\right)\right) .
\end{aligned}
$$

Then the value function at time $t$ can be expressed as

$$
J(t, w, \pi)=f\left(t, w, w ; W_{T}^{\pi}\right)+G\left(w, g\left(t, w ; W_{T}^{\pi}\right)\right) .
$$

Naturally, at the next time $t+\varepsilon$ with efficient small $\varepsilon>0$, the corresponding value function can be expressed as

$$
\begin{aligned}
J\left(t+\varepsilon, w_{\varepsilon}, \pi\right)= & f\left(t+\varepsilon, w_{\varepsilon}, w_{\varepsilon} ; W_{T}^{\pi}\right) \\
& +G\left(w_{\varepsilon}, g\left(t+\varepsilon, w_{\mathcal{\varepsilon}} ; W_{T}^{\pi}\right)\right) .
\end{aligned}
$$

Taking expectations gives us

$$
\begin{aligned}
E_{t, w} & {\left[J\left(t+\varepsilon, w_{\varepsilon}, \pi\right)\right] } \\
= & E_{t, w}\left[f\left(t+\varepsilon, w_{\varepsilon}, w_{\varepsilon} ; W_{T}^{\pi}\right)\right] \\
& \quad+E_{t, w}\left[G\left(w_{\varepsilon}, g\left(t+\varepsilon, w_{\varepsilon} ; W_{\mathrm{T}}^{\pi}\right)\right)\right] .
\end{aligned}
$$

By (6), we can construct the corresponding infinitesimal operators with respect to $J, f, G$, and $g$.

Notice that

$$
\begin{aligned}
f\left(t, w, w ; W_{T}^{\pi}\right) & =E_{t, w}\left[F\left(w, W_{T}^{\pi}\right)\right] \\
& =E_{t, w}\left[E_{t+\varepsilon, w_{\varepsilon}}\left[F\left(w, W_{T}^{\pi}\right)\right]\right] \\
& =E_{t, w}\left[f\left(t+\varepsilon, w_{\varepsilon}, w ; W_{T}^{\pi}\right)\right], \\
g\left(t, w ; W_{T}^{\pi}\right) & =E_{t, w}\left[W_{T}^{\pi}\right]=E_{t, w}\left[E_{t+\varepsilon, w_{\varepsilon}}\left[W_{T}^{\pi}\right]\right] \\
& =E_{t, w}\left[g\left(t+\varepsilon, w_{\varepsilon} ; W_{T}^{\pi}\right)\right] .
\end{aligned}
$$


Then, the value function at the time $t$ can be expressed as

$$
\begin{aligned}
J(t, w, \pi)= & E_{t, w}\left[f\left(t+\varepsilon, w_{\varepsilon}, w ; W_{T}^{\pi}\right)\right] \\
& +G\left(w, E_{t, w}\left[g\left(t+\varepsilon, w_{\varepsilon} ; W_{T}^{\pi}\right)\right]\right) .
\end{aligned}
$$

Next, we construct the infinitesimal operator $A^{\pi} J(t, w$, $\pi)$. By (6), (15), and (17), we have

$$
\begin{aligned}
A^{\pi} J(t, w, \pi)= & E_{t, w}\left[J\left(t+\varepsilon, w_{\varepsilon}, \pi\right)\right]-J(t, w, \pi) \\
= & E_{t, w}\left[f\left(t+\varepsilon, w_{\varepsilon}, w_{\varepsilon} ; W_{T}^{\pi}\right)\right] \\
& +E_{t, w}\left[G\left(w_{\varepsilon}, g\left(t+\varepsilon, w_{\varepsilon} ; W_{T}^{\pi}\right)\right)\right] \\
& -E_{t, w}\left[f\left(t+\varepsilon, w_{\varepsilon}, w ; W_{T}^{\pi}\right)\right] \\
& -G\left(w, E_{t, w}\left[g\left(t+\varepsilon, w_{\varepsilon} ; W_{T}^{\pi}\right)\right]\right) .
\end{aligned}
$$

Firstly, notice the three terms $E_{t, w}\left[f\left(t+\varepsilon, w_{\varepsilon}, w_{\varepsilon} ; W_{T}^{\pi}\right)\right]$, $E_{t, w}\left[f\left(t+\varepsilon, w_{\varepsilon}, w ; W_{T}^{\pi}\right)\right]$, and $E_{t, w}\left[G\left(w_{\varepsilon}, g\left(t+\varepsilon, w_{\varepsilon} ; W_{T}^{\pi}\right)\right)\right]$ in (18); we could construct three corresponding infinitesimal operators $A^{\pi} f\left(t, w, w ; W_{T}^{\pi}\right), A^{\pi} f^{w}\left(t, w ; W_{T}^{\pi}\right)$, and $A^{\pi} G(w$, $\left.g\left(t, w ; W_{T}^{\pi}\right)\right)$; that is,

$$
\begin{aligned}
& A^{\pi} f\left(t, w, w ; W_{T}^{\pi}\right) \\
& =E_{t, w}\left[f\left(t+\varepsilon, w_{\varepsilon}, w_{\varepsilon} ; W_{T}^{\pi}\right)\right]-f\left(t, w, w ; W_{T}^{\pi}\right), \\
& A^{\pi} f^{w}\left(t, w ; W_{T}^{\pi}\right) \\
& \quad=E_{t, w}\left[f\left(t+\varepsilon, w_{\varepsilon}, w ; W_{T}^{\pi}\right)\right]-f\left(t, w, w ; W_{T}^{\pi}\right), \\
& A^{\pi} G\left(w, g\left(t, w ; W_{T}^{\pi}\right)\right) \\
& \quad=E_{t, w}\left[G\left(w_{\varepsilon}, g\left(t+\varepsilon, w_{\varepsilon} ; W_{T}^{\pi}\right)\right)\right] \\
& \quad-G\left(w, g\left(t, w ; W_{T}^{\pi}\right)\right) .
\end{aligned}
$$

Secondly, notice the rest term $G\left(w, E_{t, w}\left[g\left(t+\varepsilon, w_{\varepsilon} ; W_{T}^{\pi}\right)\right]\right)$ in (18), which can be expressed as $G\left(w, E_{t, w}[g(t+\right.$ $\left.\left.\left.\varepsilon, w_{\varepsilon} ; W_{T}^{\pi}\right)\right]\right)=G^{w}\left(E_{t, w}\left[g\left(t+\varepsilon, w_{\varepsilon} ; W_{T}^{\pi}\right)\right]\right)$. By (11)-(12), we can construct another operator

$$
\begin{aligned}
\left(H_{g}^{\pi} G\right)\left(t, w ; E_{T}^{\pi}\right)= & G^{w}\left(E_{t, w}\left[g\left(t+\varepsilon, w_{\varepsilon} ; W_{T}^{\pi}\right)\right]\right) \\
& -G^{w}\left(g\left(t, w ; W_{T}^{\pi}\right)\right) .
\end{aligned}
$$

Therefore, by (18)-(20), the infinitesimal operator $A^{\pi} J(t, w, \pi)$ can be expressed as

$$
\begin{aligned}
A^{\pi} J(t, w, \pi)= & A^{\pi} f\left(t, w, w ; W_{T}^{\pi}\right)-A^{\pi} f^{w}\left(t, w ; W_{T}^{\pi}\right) \\
& +A^{\pi}(G \diamond g)\left(t, w ; W_{T}^{\pi}\right) \\
& -\left(H_{g}^{\pi} G\right)\left(t, w ; W_{T}^{\pi}\right) .
\end{aligned}
$$

Collecting all results and noticing the fact $A^{\widehat{\pi}} V(t, w)=$ $A^{\widehat{\pi}} J(t, w, \widehat{\pi})$ which is given in (9), we arrive at the extended $\mathrm{HJB}$ system which is consistent with Björk and Murgoci [7].
Proposition 3. The extended HJB system for the Nash equilibrium problem satisfies

$$
\begin{aligned}
& \sup _{\pi \in \Pi}\left\{A^{\pi} V(t, w)-A^{\pi} f\left(t, w, w ; W_{T}^{\pi}\right)\right. \\
& \quad+A^{\pi} f^{w}\left(t, w ; W_{T}^{\pi}\right)-A^{\pi}(G \diamond g)\left(t, w ; W_{T}^{\pi}\right) \\
& \left.\quad+\left(H_{g}^{\pi} G\right)\left(t, w ; W_{T}^{\pi}\right)\right\}=0, \quad t \in[0, T] \\
& A^{\widehat{\pi}} f^{w}\left(t, w ; W_{T}^{\pi}\right)=0, \quad t \in[0, T] \\
& A^{\widehat{\pi}} g\left(t, w ; W_{T}^{\pi}\right)=0, \quad t \in[0, T] \\
& V\left(T, w ; W_{T}^{\pi}\right)=F\left(w, W_{T}^{\pi}\right)+G\left(w, W_{T}^{\pi}\right), \\
& f^{w}\left(T, w ; W_{T}^{\pi}\right)=F\left(w, W_{T}^{\pi}\right), \\
& g\left(T, w ; W_{T}^{\pi}\right)=w .
\end{aligned}
$$

Here, $\hat{\pi}$ is the equilibrium control law which realizes the supremum in the first equation of (22), and $f^{y}, G \diamond g$, and $H_{g}^{\pi} G$ are given in (10)-(12).

Remark 4. (1) In the case when $F\left(w, W_{T}^{\pi}\right)$ does not depend on $w$, the terms are

$$
\left(A^{\pi} f\right)\left(t, w, w ; W_{T}^{\pi}\right)-\left(A^{\pi} f^{w}\right)\left(t, w ; W_{T}^{\pi}\right)=0 .
$$

(2) By the infinitesimal operator $A^{\pi} h(t, w)=E_{t, w}[h(t+$ $\left.\left.\varepsilon, w_{\varepsilon}\right)\right]-h(t, w)$ and the first-order Taylor extension $h\left(w_{0}+\right.$ $\Delta w)=h\left(w_{0}\right)+h^{\prime}\left(w_{0}\right) \Delta w+o(\Delta w)$, it is easy to see

$$
\begin{aligned}
G^{w}( & \left.E_{t, w}\left[g\left(t+\varepsilon, w_{\varepsilon} ; W_{T}^{\pi}\right)\right]\right) \\
= & G^{w}\left(g\left(t, w ; W_{T}^{\pi}\right)+A^{\pi} g\left(t, w ; W_{T}^{\pi}\right)\right) \\
& +o\left(A^{\pi} g\left(t, w ; W_{T}^{\pi}\right)\right) \\
= & G^{w}\left(g\left(t, w ; W_{T}^{\pi}\right)\right) \\
& +G_{y}^{w}\left(g\left(t, w ; W_{T}^{\pi}\right)\right) A^{\pi}\left(g\left(t, w ; W_{T}^{\pi}\right)\right) \\
& +o\left(A^{\pi} g\right),
\end{aligned}
$$

where $G_{y}^{w}(y)=\left(\partial G^{w} / \partial y\right)(y)$.

(3) A simple calculation shows that (see Björk and Murgoci [7])

$$
\begin{aligned}
- & A^{\pi}\left[G\left(g\left(t, w ; W_{T}^{\pi}\right)\right)\right] \\
& +G^{\prime}\left(g\left(t, w ; W_{T}^{\pi}\right)\right) A^{\pi}\left(g\left(t, w ; W_{T}^{\pi}\right)\right) \\
& =-\frac{1}{2} \sigma^{2}(t, w, \pi) G^{\prime \prime}\left(g\left(t, w ; W_{T}^{\pi}\right)\right) g_{w}^{2}\left(t, w ; W_{T}^{\pi}\right) .
\end{aligned}
$$

(4) In the extended HJB system, the partial derivatives of $f$ and $g$ should be evaluated at $\left(t, w, w ; W_{T}^{\pi}\right)$ and $\left(t, w ; W_{T}^{\pi}\right)$, respectively. 


\section{Corporate International Investment Problem with Mean-Variance Criterion}

3.1. The Market Setting. Let $(\Omega, \mathscr{F}, \mathbf{P})$ be a probability space equipped with a filtration $\mathbf{F}=\left(\mathscr{F}_{t}\right)_{0 \leq t \leq T}$ satisfying the usual conditions; that is, $\left(\mathscr{F}_{t}\right)_{0 \leq t \leq T}$ is right-continuous and $\mathbf{P}$-complete, where $T$ is a positive finite constant representing the time horizon. Suppose that all stochastic processes and random variables are defined on the filtered probability space $(\Omega, \mathscr{F}, \mathbf{F}, \mathbf{P})$.

3.2. Corporate International Investment Problem (CIIP). The purpose of this paper is to study corporate international investment problem (CIIP) that a firm invests in both bank and real project which contains two markets: home market and foreign market. We consider a risk-free asset with dynamic price process

$$
d S_{0}(t)=r S_{0}(t) d t
$$

where $r$ is constant short rate.

Choi [32] supposed a "two-country" firm whose static cash flows for time $t$ from its home and foreign operations could be written in a standard way

$$
\begin{aligned}
& R_{t}=\left(P_{t}-C_{t}\right) Q_{t}, \\
& R_{t}^{*}=e_{t}\left(P_{t}^{*}-C_{t}^{*}\right) Q_{t}^{*},
\end{aligned}
$$

where $P_{t}\left(P_{t}^{*}\right)$ and $C_{t}\left(C_{t}^{*}\right)$ were uncertain output and input prices in home (foreign) market, $Q_{t}\left(Q_{t}^{*}\right)$ was the quantity of output which was assumed to be certain, and $e_{t}$ is uncertain exchange rate.

Contrary to the model of Choi [32], Bellalah and $\mathrm{Wu}$ [33] took the attitude that the quantity of output was a decreasing function of the output price, and the cash flows of firm's activity were taxed at a certain rate. Thus they considered that a "two-country" firm's static cash flows from its home $\left(R_{t}\right)$ and foreign $\left(R_{t}^{*}\right)$ operations could be written in the following form:

$$
\begin{aligned}
& R_{t}=(1-\tau)\left(P_{t}-C_{t}\right) Q_{t}, \quad Q_{t}=P_{t}^{\beta}, \\
& R_{t}^{*}=e_{t}\left(1-\tau^{*}\right)\left(P_{t}^{*}-C_{t}^{*}\right) Q_{t}^{*}, \quad Q_{t}^{*}=\left(P_{t}^{*}\right)^{\beta^{*}},
\end{aligned}
$$

where the terms $\beta$ and $\beta^{*}$ are negative constants. $\tau$ and $\tau^{*}$ are tax rates in home and foreign market, respectively.

Similar to Bellalah and $\mathrm{Wu}$ [33], we assume the following dynamics for output price, input price, and exchange rate:

$$
\begin{aligned}
& \frac{d P_{t}}{P_{t}}=\mu_{p} d t+\sigma d B_{t}^{1}, \\
& \frac{d C_{t}}{C_{t}}=\mu_{c} d t+\sigma d B_{t}^{1}, \\
& \frac{d P_{t}^{*}}{P_{t}^{*}}=\mu_{p^{*}} d t+\sigma^{*} d B_{t}^{2},
\end{aligned}
$$

$$
\begin{aligned}
\frac{d C_{t}^{*}}{C_{t}^{*}} & =\mu_{c^{*}} d t+\sigma^{*} d B_{t}^{2}, \\
\frac{d e_{t}}{e_{t}} & =\left(\mu_{e}+\lambda_{e}\right) d t+\sigma_{e} d B_{t}^{3} .
\end{aligned}
$$

The initial values of above variables are $P_{0}, C_{0}, P_{0}^{*}, C_{0}^{*}$, and $e_{0}$, respectively. The terms $\mu_{p}, \mu_{p^{*}}, \mu_{c}, \mu_{c^{*}}$, and $\mu_{e}$ are bounded constants which represent the instantaneous expected rates of these variables, respectively. $\lambda_{e}$ reflects the information cost carried by managers in order to get information about foreign exchange markets. The terms $\sigma, \sigma^{*}$, and $\sigma_{e}$ are the instantaneous volatility coefficients in home, foreign, and exchange market. The terms $B_{t}^{1}, B_{t}^{2}$, and $B_{t}^{3}$ are three onedimensional mutually dependent Brownian motions which represent the external sources of uncertainty in the markets with correlation coefficients $\rho_{12}, \rho_{13}$, and $\rho_{23}$.

The changes in the cash flows from home market can be written as (see Appendix A)

$$
\frac{d R_{t}}{R_{t}}=\widehat{f}(t) d t+(\beta+1) \sigma d B_{t}^{1},
$$

where

$$
\begin{aligned}
\widehat{f}(t)= & \frac{1}{2}(\beta+1)^{2} \sigma^{2}+(\beta+1)\left(\mu_{p}-\frac{1}{2} \sigma^{2}\right) \\
& +\frac{\left(\mu_{p}-\mu_{c}\right) C_{0} e^{\mu_{c} t}}{P_{0} e^{\mu_{p} t}-C_{0} e^{\mu_{c} t}} .
\end{aligned}
$$

However, managers should pay sunk costs in order to obtain information. Therefore, they require an additional return to get compensation for their resources engaged in the search for information. Information costs have the dimension of incremental rates of return which are added to the drift in the process describing the dynamics for different variables. So, the real changes in the cash flows from home market should be written as

$$
\begin{aligned}
\frac{d R_{t}}{R_{t}} & =\left[\hat{f}(t)+\lambda_{R}\right] d t+(\beta+1) \sigma d B_{t}^{1}, \\
R_{0} & =(1-\tau) P_{0}^{\beta}\left(P_{0}-C_{0}\right),
\end{aligned}
$$

where $\lambda_{R}$ is the information costs rate in home market.

Similarly, the expression of changes in the cash flows from foreign market before exchanging can be written as

$$
\frac{d \bar{R}_{t}}{\bar{R}_{t}}=\bar{f}(t) d t+\left(\beta^{*}+1\right) \sigma^{*} d B_{t}^{2},
$$

where

$$
\begin{aligned}
\bar{f}(t)= & \frac{1}{2}\left(\beta^{*}+1\right)^{2}\left(\sigma^{*}\right)^{2} \\
& +\left(\beta^{*}+1\right)\left(\mu_{p^{*}}-\frac{1}{2}\left(\sigma^{*}\right)^{2}\right) \\
& +\frac{\left(\mu_{p^{*}}-\mu_{c^{*}}\right) C_{0}^{*} e^{\mu_{c} t}}{P_{0}^{*} e^{\mu_{p^{*}} t}-C_{0}^{*} e^{\mu_{c^{*}} t}} .
\end{aligned}
$$


Then, from $R^{*}=e_{t} \bar{R}_{t}$ and (32) and (33), the expression of changes in the cash flows from foreign market after exchanging is given by

$$
\begin{aligned}
\frac{d R_{t}^{*}}{R_{t}^{*}}= & {\left[\widehat{f}^{*}(t)+\mu_{e}+\lambda_{e}\right] d t+\left(\beta^{*}+1\right) \sigma^{*} d B_{t}^{2} } \\
& +\sigma_{e} d B_{t}^{3}
\end{aligned}
$$

where

$$
\widehat{f}^{*}(t)=\bar{f}(t)+\left(\beta^{*}+1\right) \sigma^{*} \sigma_{e} \rho_{23} .
$$

However, managers also should pay sunk costs in order to obtain information in foreign market. Thus, they also require an additional return to get compensation for their resources engaged in the search for information. So the real changes in the cash flows from foreign market can be expressed as

$$
\begin{aligned}
\frac{d R_{t}^{*}}{R_{t}^{*}}= & {\left[\widehat{f}^{*}(t)+\lambda_{R^{*}}+\mu_{e}+\lambda_{e}\right] d t+\left(\beta^{*}+1\right) \sigma^{*} d B_{t}^{2} } \\
& +\sigma_{e} d B_{t}^{3} \\
R_{0}^{*}= & e_{0}\left(1-\tau^{*}\right) P_{0}^{\beta^{*}}\left(P_{0}^{*}-C_{0}^{*}\right) .
\end{aligned}
$$

3.3. The Wealth Process. Suppose that $W_{t}^{\pi}$ is the corporate wealth at time $t \geq 0$. Let $\pi_{1}(t)$ represent the money invested in home market and $\pi_{2}(t)$ represent those invested in foreign market. Then, the rest money $W_{t}^{\pi}-\pi_{1}(t)-\pi_{2}(t)$ is invested in home money account.
From (32), (37), and bank account, we know that the corporate wealth can be written as

$$
\begin{gathered}
d W_{t}^{\pi}=\left[\pi_{1}\left(\widehat{f}^{*}(t)-r+\lambda_{R^{*}}+\mu_{e}+\lambda_{e}\right)\right. \\
\left.+\pi_{2}\left(\widehat{f}(t)-r+\lambda_{R}\right)+W_{t}^{\pi} r\right] d t+\pi_{1}(\beta+1) \\
\cdot \sigma d B_{t}^{1}+\pi_{2}\left(\beta^{*}+1\right) \sigma^{*} d B_{t}^{2}+\pi_{2} \sigma_{e} d B_{t}^{3} .
\end{gathered}
$$

We assume that the initial value of corporate wealth $W_{0}>$ 0 and $\pi=\left(\pi_{1}, \pi_{2}\right)$ is a locally measurable bounded process, almost surely.

Definition 5. A strategy $\pi=\left(\pi_{1}, \pi_{2}\right)_{0 \leq t \leq T}$ is said to be admissible if it satisfies the following conditions:

(i) Stochastic process $\pi=\left(\pi_{1}, \pi_{2}\right)_{0 \leq t \leq T}$ is $\mathscr{F}_{t^{-}}$ progressively measurable

(ii) $E\left[\int_{0}^{T}\left(\pi_{1}^{2}+\pi_{2}^{2}\right) d t\right]<+\infty$

(iii) The wealth process in (38) has a unique solution $W_{t}^{\pi}$ on $[0, T]$

For convenience, we will write $W_{t}^{\pi}$ as $W_{t}$ in next section.

Next, we consider the CIIP with constant risk aversion coefficient and state-dependent risk aversion coefficient, respectively.

3.4. Corporate International Investment Problem (CIIP) with Constant Risk Aversion Coefficient. In this section, we consider the mean-variance model for corporate international investment problem:

$$
\begin{aligned}
\max _{\left\{\pi_{t}\right\} \in \Pi} E_{t, w}\left[W_{T}^{\pi}\right]-\frac{\gamma}{2} \operatorname{Var}_{t, w}\left[W_{T}^{\pi}\right] \\
\text { s.t. } \quad d W_{t} \\
=\left[\pi_{1}\left(\hat{f}^{*}(t)-r+\lambda_{R^{*}}+\mu_{e}+\lambda_{e}\right)+\pi_{2}\left(\hat{f}(t)-r+\lambda_{R}\right)+W_{t} r\right] d t+\pi_{1}(\beta+1) \sigma d B_{t}^{1}+\pi_{2}\left(\beta^{*}+1\right) \sigma^{*} d B_{t}^{2} \\
+\pi_{2} \sigma_{e} d B_{t}^{3},
\end{aligned}
$$

where $W_{t}$ is the corporate wealth at time $t>0, E_{t, w}$ is the expected value operator under $W_{t}=w$ conditions, and $\operatorname{Var}_{t, w}$ is the variance operator under $W_{t}=w$ conditions. The terms $\pi_{1}, \pi_{2}$ are the amounts of corporate capital budget invested in home and foreign market, respectively. And $\gamma$ is the constant risk aversion coefficient which leads to aggressive or passive corporate investments. The primary motive of an aggressive company, which is usually a leader in the industry and a risk-taker, is to anticipate greater expected return for investing in foreign market. However, the motivation of a passive company, which is a follower and risk-avoider, is to maintain its existing market position rather than to increase it. Generally, this passive company reacts to the leader when the chance of foreign investment has been "proven" (see Choi [32]).
Denote the value functional by

$$
J(t, w, \pi)=E_{t, w}\left[W_{T}^{\pi}\right]-\frac{\gamma}{2} \operatorname{Var}_{t, w}\left[W_{T}^{\pi}\right] .
$$

The object is to maximize expected return with a risk penalty. According to the definition of conditional variance

$$
\operatorname{Var}_{t, w}\left[W_{T}\right]=E_{t, w}\left[W_{T}^{2}\right]-E_{t, w}^{2}\left[W_{T}\right]
$$

we can rewrite our value functional as

$$
\begin{aligned}
J(t, w, \pi) & =E_{t, w}\left[W_{T}^{\pi}\right]-\frac{\gamma}{2} \operatorname{Var}_{t, w}\left[W_{T}^{\pi}\right] \\
& =E_{t, w}\left[W_{T}^{\pi}\right]
\end{aligned}
$$




$$
\begin{aligned}
& -\frac{\gamma}{2}\left(E_{t, w}\left[\left(W_{T}^{\pi}\right)^{2}\right]-E_{t, w}^{2}\left[W_{T}^{\pi}\right]\right) \\
= & E_{t, w}\left[W_{T}^{\pi}-\frac{\gamma}{2}\left(W_{T}^{\pi}\right)^{2}\right]+\frac{\gamma}{2}\left(E_{t, w}\left[W_{T}^{\pi}\right]\right)^{2} \\
:= & E_{t, w}\left[F\left(W_{T}^{\pi}\right)\right]+G\left(E_{t, w}\left[W_{T}^{\pi}\right]\right),
\end{aligned}
$$

where $F(x)=x-(\gamma / 2) x^{2}$ and $G(x)=(\gamma / 2) x^{2}$.

From Proposition 3 and (1)-(3) in Remark 4, the extended HJB system is then given by the following system.

Proposition 6. The extended HJB system for the Nash equilibrium problem satisfies

$$
\begin{aligned}
\sup _{\pi \in \Pi} & \left\{A^{\pi} V(t, w)\right. \\
& \left.-\frac{1}{2} \sigma^{2}(w, \pi) G^{\prime \prime}\left(g\left(t, w ; W_{T}^{\pi}\right)\right) g_{w}^{2}\left(t, w ; W_{T}^{\pi}\right)\right\}
\end{aligned}
$$

$$
=0 \text {, }
$$

$V(T, w)=w$,

$$
\begin{aligned}
& A^{\widehat{\pi}} g\left(t, w ; W_{T}^{\widehat{\pi}}\right)=0, \\
& g\left(T, w ; W_{T}^{\widehat{\pi}}\right)=w,
\end{aligned}
$$

where $\hat{\pi}$ is the equilibrium control law which realizes the supremum in the first equation of (43).

Furthermore, we have the following HJB system for corporate international investment problem (CIIP).

Proposition 7. The extended HJB system for CIIP satisfies

$$
\begin{aligned}
\max _{\{\pi \in \Pi\}} & \left\{V_{t}+V_{W} W \Delta_{u}^{1}+\frac{1}{2}\left(V_{W W}-\gamma g_{w}^{2}\right) W^{2} \Delta_{d}^{1}+\pi\left[V_{W} W\left(\Delta_{u}^{2}-\Delta_{u}^{1}\right)+\frac{1}{2}\left(V_{W W}-\gamma g_{w}^{2}\right) W^{2} \Delta_{d}^{2}\right]+\frac{1}{2} \pi^{2}\left(V_{W W}-\gamma g_{w}^{2}\right) W^{2} \Delta_{d}^{3}\right\} \\
& =0 \\
& V(T, w)=w \\
& A^{\widehat{u}} g\left(t, w ; W_{T}^{\hat{\pi}}\right)=0, \\
& g\left(T, w ; W_{T}^{\widehat{\pi}}\right)=w,
\end{aligned}
$$

where

$$
\begin{aligned}
& \Delta_{u}^{1}=\widehat{f}^{*}(t)-r+\lambda_{R^{*}}+\mu_{e}+\lambda_{e}, \\
& \Delta_{u}^{2}=\widehat{f}(t)-r+\lambda_{R}, \\
& \Delta_{d}^{1}=(\beta+1)^{2} \sigma^{2}, \\
& \Delta_{d}^{2}=(\beta+1)\left(\beta^{*}+1\right) \sigma \sigma^{*} \rho_{12}+(\beta+1) \sigma \sigma_{e} \rho_{13}, \\
& \Delta_{d}^{3}=\left(\beta^{*}+1\right)^{2}\left(\sigma^{*}\right)^{2}+\sigma_{e}^{2}+2\left(\beta^{*}+1\right) \sigma^{*} \sigma_{e} \rho_{23},
\end{aligned}
$$

and $\hat{\pi}$ is the equilibrium control law which realizes the supremum in the first equation of (44).

The term $V_{t}$ describes the growth rate of the manager's value function. The term $V_{W}$ describes the marginal increase of stochastic return. The term $V_{W W}$ reflects the effects of stochastic return. The term $\Delta_{d}^{1}=(\beta+1)^{2} \sigma^{2}$ represents the home returns volatility. The term $(\beta+1)\left(\beta^{*}+1\right) \sigma \sigma^{*} \rho_{12}$ reflects the home and foreign returns correlation volatility. And the term $(\beta+1) \sigma \sigma_{e} \rho_{13}$ shows the home returns and exchange rate correlation volatility. Therefore, the term $\Delta_{d}^{2}$ represents the sum of these two correlation volatilities. The term $\Delta_{d}^{3}=$ $\left(\beta^{*}+1\right)^{2}\left(\sigma^{*}\right)^{2}+\sigma_{e}^{2}+2\left(\beta^{*}+1\right) \sigma^{*} \sigma_{e} \rho_{23}$ represents the sum of foreign returns volatility, exchange rate, and their correlation volatility.
By the optimal condition of (44), we can deduce an optimal strategy. Further, by conjecturing the form of value function and incorporating the optimal strategy into extended HJB system, we can obtain the value function.

Theorem 8. The equilibrium time-consistent strategy for corporate international investment problem (CIIP) is of the following forms:

$$
\begin{aligned}
& \pi_{1}^{*}(t)=\frac{1}{\gamma} e^{r(T-t)} \Delta_{m}^{1}, \\
& \pi_{2}^{*}(t)=\frac{1}{\gamma} e^{r(T-t)} \Delta_{m}^{2} .
\end{aligned}
$$

And the value functions $V$ and $g$ are of the following forms:

$$
\begin{aligned}
& V(t, w)=e^{r(T-t)} w+\frac{1}{\gamma} \int_{t}^{T}[\Lambda(s)-\Gamma(s)] d s, \\
& g(t, w)=e^{r(T-t)} w+\frac{1}{\gamma} \int_{t}^{T} \Lambda(s) d s,
\end{aligned}
$$

where

$$
\begin{aligned}
& \Lambda(t)=\Delta_{m}^{1} \Delta_{u}^{1}+\Delta_{m}^{2} \Delta_{u}^{2}, \\
& \Gamma(t)=-\frac{1}{2}\left(\Delta_{m}^{1}\right)^{2} \Delta_{d}^{1}-\Delta_{m}^{1} \Delta_{m}^{2} \Delta_{d}^{2}-\frac{1}{2}\left(\Delta_{m}^{2}\right)^{2} \Delta_{d}^{3},
\end{aligned}
$$


the terms $\Delta_{\mathcal{u}}^{1}, \Delta_{u}^{2}, \Delta_{d}^{1}, \Delta_{d}^{2}$, and $\Delta_{d}^{3}$ are given in (45), and

$$
\begin{aligned}
& \Delta_{m}^{1}=\frac{\Delta_{u}^{1} \Delta_{d}^{3}-\Delta_{u}^{2} \Delta_{d}^{2}}{\Delta_{d}^{1} \Delta_{d}^{3}-\left(\Delta_{d}^{2}\right)^{2}}, \\
& \Delta_{m}^{2}=\frac{\Delta_{u}^{2} \Delta_{d}^{1}-\Delta_{u}^{1} \Delta_{d}^{2}}{\Delta_{d}^{1} \Delta_{d}^{3}-\left(\Delta_{d}^{2}\right)^{2}} .
\end{aligned}
$$

The proof is given in Appendix B.

If we denote that $M=(1 / \gamma)\left(1 /\left(\Delta_{d}^{1} \Delta_{d}^{3}-\left(\Delta_{d}^{2}\right)^{2}\right)\right) e^{r(T-t)}$ and by (46), we have

$$
\begin{aligned}
& \frac{\partial \pi_{1}^{*}}{\partial \lambda_{R}}=-M \Delta_{d}^{2}, \\
& \frac{\partial \pi_{1}^{*}}{\partial \lambda_{R^{*}}}=M \Delta_{d}^{3}, \\
& \frac{\partial \pi_{1}^{*}}{\partial \mu_{e}}=M \Delta_{d}^{3}, \\
& \frac{\partial \pi_{1}^{*}}{\partial \lambda_{e}}=M \Delta_{d}^{3}, \\
& \frac{\partial \pi_{2}^{*}}{\partial \lambda_{R}}=M \Delta_{d}^{1}, \\
& \frac{\partial \pi_{2}^{*}}{\partial \lambda_{R^{*}}}=-M \Delta_{d}^{2}, \\
& \frac{\partial \pi_{2}^{*}}{\partial \mu_{e}}=-M \Delta_{d}^{2}, \\
& \frac{\partial \pi_{2}^{*}}{\partial \lambda_{e}}=-M \Delta_{d}^{2} .
\end{aligned}
$$

Note that $\Delta_{d}^{i}>0, i=1,2,3$, so we can get the following arguments.

Remark 9. If $M \geq 0, \pi_{1}^{*}$ increases with respect to foreign market information cost $\lambda_{R^{*}}$, exchange market information $\operatorname{cost} \lambda_{e}$, and exchange rate $\mu_{e}$ and decreases with respect to home market information cost $\lambda_{R} \cdot \pi_{2}^{*}$ decreases with respect to $\lambda_{R^{*}}, \lambda_{e}$, and $\mu_{e}$ and increases with respect to $\lambda_{R}$ and vice versa.

Now, we explain the economic meanings of the equilibrium time-consistent strategy. By (49), we have

$$
\begin{aligned}
\Delta_{m}^{1} & =\frac{1}{\Delta_{d}^{1} \Delta_{d}^{3}-\left(\Delta_{d}^{2}\right)^{2}}\left[\Delta_{d}^{3}\left(\lambda_{R^{*}}+\lambda_{e}\right)-\Delta_{d}^{2} \lambda_{R}\right. \\
& +\mu_{e} \Delta_{d}^{3}-r\left(\Delta_{d}^{3}-\Delta_{d}^{2}\right) \\
& +\Delta_{d}^{3} \mu_{p^{*}}\left(\frac{P_{0}^{*} e^{\mu_{p^{*}} t}}{P_{0}^{*} e^{\mu_{p^{*}} t}-C_{0}^{*} e^{\mu_{c}^{*} t}}+\beta^{*}\right) \\
& -\Delta_{d}^{2} \mu_{p}\left(\frac{P_{0} e^{\mu_{p} t}}{P_{0} e^{\mu_{p} t}-C_{0} e^{\mu_{c} t}}+\beta\right)
\end{aligned}
$$

$$
\begin{aligned}
& +\Delta_{d}^{2} \mu_{c} \frac{C_{0} e^{\mu_{c} t}}{P_{0} e^{\mu_{p} t}-C_{0} e^{\mu_{c} t}}-\Delta_{d}^{3} \mu_{c^{*}} \frac{C_{0}^{*} e^{\mu_{c}^{*} t}}{P_{0}^{*} e^{\mu_{p^{*}} t}-C_{0}^{*} e^{\mu_{c} * t}} \\
& +\frac{1}{2} \Delta_{d}^{3}\left(\beta^{*}\left(\beta^{*}+1\right)\left(\sigma^{*}\right)^{2}+2\left(\beta^{*}+1\right) \sigma^{*} \sigma_{e} \rho_{23}\right) \\
& \left.-\frac{1}{2} \Delta_{d}^{2} \beta(\beta+1) \sigma^{2}\right],
\end{aligned}
$$$$
\Delta_{m}^{2}=-\frac{1}{\Delta_{d}^{1} \Delta_{d}^{3}-\left(\Delta_{d}^{2}\right)^{2}}\left[\Delta_{d}^{2}\left(\lambda_{R^{*}}+\lambda_{e}\right)-\Delta_{d}^{1} \lambda_{R}\right.
$$$$
+\mu_{e} \Delta_{d}^{2}-r\left(\Delta_{d}^{2}-\Delta_{d}^{1}\right)
$$$$
+\Delta_{d}^{2} \mu_{p^{*}}\left(\frac{P_{0}^{*} e^{\mu_{p^{*}} t}}{P_{0}^{*} e^{\mu_{p^{*}}}-C_{0}^{*} e^{\mu_{c}^{* t}}}+\beta^{*}\right)
$$$$
-\Delta_{d}^{1} \mu_{p}\left(\frac{P_{0} e^{\mu_{p} t}}{P_{0} e^{\mu_{p} t}-C_{0} e^{\mu_{c} t}}+\beta\right)
$$$$
+\Delta_{d}^{1} \mu_{c} \frac{C_{0} e^{\mu_{c} t}}{P_{0} e^{\mu_{p} t}-C_{0} e^{\mu_{c} t}}-\Delta_{d}^{2} \mu_{c^{*}} \frac{C_{0}^{*} e^{\mu_{c} t}}{P_{0}^{*} e^{\mu_{p^{*}} t}-C_{0}^{*} e^{\mu_{c^{*}} t}}
$$$$
+\frac{1}{2} \Delta_{d}^{2}\left(\beta^{*}\left(\beta^{*}+1\right)\left(\sigma^{*}\right)^{2}+2\left(\beta^{*}+1\right) \sigma^{*} \sigma_{e} \rho_{23}\right)
$$$$
\left.-\frac{1}{2} \Delta_{d}^{1} \beta(\beta+1) \sigma^{2}\right] \text {. }
$$

For simplifying, we denote that

$$
\begin{aligned}
H_{1}^{1}= & \Delta_{d}^{3}\left(\lambda_{R^{*}}+\lambda_{e}\right)-\Delta_{d}^{2} \lambda_{R}+\mu_{e} \Delta_{d}^{3}-r\left(\Delta_{d}^{3}-\Delta_{d}^{2}\right) \\
& +\Delta_{d}^{3} \mu_{p^{*}}\left(\frac{P_{0}^{*} e^{\mu_{p^{*}} t}}{P_{0}^{*} e^{\mu_{p^{*}} t}-C_{0}^{*} e^{\mu_{c^{*}} t}}+\beta^{*}\right) \\
& -\Delta_{d}^{2} \mu_{p}\left(\frac{P_{0} e^{\mu_{p} t}}{P_{0} e^{\mu_{p} t}-C_{0} e^{\mu_{c} t}}+\beta\right) \\
& +\Delta_{d}^{2} \mu_{c} \frac{C_{0} e^{\mu_{c} t}}{P_{0} e^{\mu_{p} t}-C_{0} e^{\mu_{c} t}} \\
& -\Delta_{d}^{3} \mu_{c^{*}} \frac{C_{0}^{*} e^{\mu_{c}^{*} t}}{P_{0}^{*} e^{\mu_{p^{*}} t}-C_{0}^{*} e^{\mu_{c}^{*} t}}, \\
H_{1}^{2}= & \frac{1}{2} \Delta_{d}^{3}\left(\beta^{*}\left(\beta^{*}+1\right)\left(\sigma^{*}\right)^{2}+2\left(\beta^{*}+1\right) \sigma^{*} \sigma_{e} \rho_{23}\right) \\
& -\frac{1}{2} \Delta_{d}^{2} \beta(\beta+1) \sigma^{2}, \\
H_{2}^{1}= & \Delta_{d}^{2}\left(\lambda_{R^{*}}+\lambda_{e}\right)-\Delta_{d}^{1} \lambda_{R}+\mu_{e} \Delta_{d}^{2}-r\left(\Delta_{d}^{2}-\Delta_{d}^{1}\right) \\
& +\Delta_{d}^{2} \mu_{p^{*}}\left(\frac{P_{0}^{*} e^{\mu_{p^{*}} t}}{P_{0}^{*} e^{\mu_{p^{*}} t}-C_{0}^{*} e^{\mu_{c}^{*} t}+\beta^{*}}\right) \\
& \Delta_{d}^{1} \mu_{p}\left(\frac{P_{0} e^{\mu_{p} t}}{P_{0} e^{\mu_{p} t}-C_{0} e^{\mu_{c} t}}+\beta\right) \\
&
\end{aligned}
$$




$$
\begin{aligned}
& +\Delta_{d}^{1} \mu_{c} \frac{C_{0} e^{\mu_{c} t}}{P_{0} e^{\mu_{p} t}-C_{0} e^{\mu_{c} t}} \\
& -\Delta_{d}^{2} \mu_{c^{*}} \frac{C_{0}^{*} e^{\mu_{c} * t}}{P_{0}^{*} e^{\mu_{p^{*} *}}-C_{0}^{*} e^{\mu_{c} * t}}, \\
H_{2}^{2}= & \frac{1}{2} \Delta_{d}^{2}\left(\beta^{*}\left(\beta^{*}+1\right)\left(\sigma^{*}\right)^{2}+2\left(\beta^{*}+1\right) \sigma^{*} \sigma_{e} \rho_{23}\right) \\
& -\frac{1}{2} \Delta_{d}^{1} \beta(\beta+1) \sigma^{2} .
\end{aligned}
$$

Then, the equilibrium time-consistent strategy in (46) can be expressed as

$$
\begin{aligned}
& \pi_{1}^{*}(t)=M\left(H_{1}^{1}+H_{1}^{2}\right), \\
& \pi_{2}^{*}(t)=-M\left(H_{2}^{1}+H_{2}^{2}\right) .
\end{aligned}
$$

The terms $M H_{1}^{1}$ and $-M H_{2}^{1}$ are referred to as the "speculative demand" or the aggressive demand. The terms $M H_{1}^{2}$ and $-\mathrm{MH}_{2}^{2}$ are called the "hedging demand." They describe the demand for home and foreign investments on risk-hedged basis, respectively.

These expressions of the speculative demand involve some foreign direct investment theories, some of which emphasize the cost advantage of host countries and advance the comparative cost savings offered by foreign production vis-a-vis home production. These are reflected in the terms $\Delta_{d}^{2} \mu_{c}\left(C_{0} e^{\mu_{c} t} /\left(P_{0} e^{\mu_{p} t}-C_{0} e^{\mu_{c} t}\right)\right)-\Delta_{d}^{3} \mu_{c^{*}}\left(C_{0}^{*} e^{\mu_{c^{*}} t} /\left(P_{0}^{*} e^{\mu_{p^{*}} t}-\right.\right.$ $\left.\left.C_{0}^{*} e^{\mu_{c} * t}\right)\right)$ in $H_{1}^{1}$ and $\Delta_{d}^{1} \mu_{c}\left(C_{0} e^{\mu_{c} t} /\left(P_{0} e^{\mu_{p} t}-C_{0} e^{\mu_{c} t}\right)\right)$ $\Delta_{d}^{2} \mu_{c^{*}}\left(C_{0}^{*} e^{\mu_{c^{*} t} t} /\left(P_{0}^{*} e^{\mu_{p^{*}} t}-C_{0}^{*} e^{\mu_{c^{*}} t}\right)\right)$ in $H_{2}^{1}$. Other foreign direct investment theories, however, incorporate the demand side effect. In this case, foreign investment depends on whether output price is higher in foreign market than in home market, which is reflected in the terms $\Delta_{d}^{3} \mu_{p^{*}}\left(P_{0}^{*} e^{\mu_{p^{*}} t} /\left(P_{0}^{*} e^{\mu_{p^{*}} t}-\right.\right.$ $\left.\left.C_{0}^{*} e^{\mu_{c}^{*} t}\right)+\beta^{*}\right)-\Delta_{d}^{2} \mu_{p}\left(P_{0} e^{\mu_{p} t} /\left(P_{0} e^{\mu_{p} t}-C_{0} e^{\mu_{c} t}\right)+\beta\right)$ in $H_{1}^{1}$ and $\Delta_{d}^{2} \mu_{p^{*}}\left(P_{0}^{*} e^{\mu_{p^{*}} t} /\left(P_{0}^{*} e^{\mu_{p^{*}} t}-C_{0}^{*} e^{\mu_{c^{*}} t}\right)+\beta^{*}\right)-$ $\Delta_{d}^{1} \mu_{p}\left(P_{0} e^{\mu_{p} t} /\left(P_{0} e^{\mu_{p} t}-C_{0} e^{\mu_{c} t}\right)+\beta\right)$ in $H_{2}^{1}$. The investment flow is also affected by the exchange rate and the domestic short rate. The effect of exchange rate can be seen in the terms $\mu_{e} \Delta_{d}^{3}$ in $H_{1}^{1}$ and $\mu_{e} \Delta_{d}^{2}$ in $H_{2}^{1}$. The effect of short rate can be seen in the terms $-r\left(\Delta_{d}^{3}-\Delta_{d}^{2}\right)$ in $H_{1}^{1}$ and $-r\left(\Delta_{d}^{2}-\Delta_{d}^{1}\right)$ in $H_{2}^{1}$.

We also take into account information costs as well as the degree of competition in home and foreign markets. In fact, if managers do not know about the foreign investment opportunity or they do not want to spend much information cost on foreign and exchange market, they will choose to invest in home market which is referred to as the "home bias puzzle." Therefore, information plays an important role in foreign investment. It can help managers to make decisions about investment in foreign market. These effects can be seen in the terms $\Delta_{d}^{3}\left(\lambda_{R^{*}}+\lambda_{e}\right)-\Delta_{d}^{2} \lambda_{R}$ in $H_{1}^{1}$ and $\Delta_{d}^{2}\left(\lambda_{R^{*}}+\lambda_{e}\right)-$ $\Delta_{d}^{1} \lambda_{R}$ in $H_{2}^{1}$.

The hedging demand describes a portfolio story as in Choi [32] and Bellalah and $\mathrm{Wu}$ [33]. If the variability of home returns is higher and the correlation between home and foreign returns is lower, managers will increase the foreign investments. In this case, the investment flow is affected by the cross-covariances and the degree of competition in home and foreign markets. These effects are reflected in the terms $H_{1}^{2}$ and $H_{2}^{2}$.

Remark 10. We find that the optimal investment strategy does not depend on the wealth process $W_{t}^{\pi^{*}}$.

Inspired by Zeng et al. [20], we can derive the relationship between conditional expectation and conditional variance of terminal wealth for corporate international investment problem (CIIP).

Theorem 11. The efficient frontier of problem (39) at initial point $\left(t, W_{t}\right)$ is given by

$$
\begin{gathered}
E_{t, w}\left[W_{T}^{\pi^{*}}\right]=e^{r(T-t)} W_{t}-\sqrt{\operatorname{Var}_{t, w}\left[W_{T}^{\pi^{*}}\right]} \\
\cdot \frac{\int_{t}^{T} \Lambda(s) d s}{\sqrt{2 \int_{t}^{T} \Gamma(s) d s}},
\end{gathered}
$$

where $\Lambda(t)$ and $\Gamma(t)$ are given in (48).

The proof is given in Appendix C.

Remark 12. For all $\left(t, W_{t}\right) \in[0, T] \times \mathscr{W}$, the relationship between $E_{t, w}\left[W_{T}^{\pi^{*}}\right]$ and $\operatorname{Var}_{t, w}\left[W_{T}^{\pi^{*}}\right]$ is given by (54). It is also called the efficient frontier of problem (39) at initial point $\left(t, W_{t}\right)$ in the modern portfolio theory.

3.5. Corporate International Investment Problem with StateDependent Risk Aversion Coefficient. In this section, we consider corporate international investment problem (CIIP) under mean-variance criterion with state-dependent risk aversion coefficient which is discussed in Björk et al. [37]; that is, the value functional is given by

$$
J(t, w, \pi)=E_{t, w}\left[W_{T}^{\pi}\right]-\frac{\gamma(w)}{2} \operatorname{Var}_{t, w}\left[W_{T}^{\pi}\right],
$$

where $\gamma(w)=\gamma / w$.

The target of manager is to find the equilibrium timeconsistent strategy or optimal solution $\pi^{*}=\left(\pi_{1}^{*}, \pi_{2}^{*}\right)$ and the equilibrium value function

$$
V(t, w)=J\left(t, w, \pi^{*}\right) .
$$

As indicated in Section 2.2, for these questions, value function (55) can be written as

$$
J(t, w, \pi)=E_{t, w}\left[F\left(w, W_{T}^{\pi}\right)\right]+G\left(w, E_{t, w}\left[W_{T}^{\pi}\right]\right),
$$

where

$$
\begin{aligned}
& F(x, y)=y-\frac{\gamma}{2 x} y^{2}, \\
& G(x, y)=\frac{\gamma}{2 x} y^{2} .
\end{aligned}
$$


Note that

$$
\begin{aligned}
V(t, w) & =J\left(t, w, \pi^{*}\right) \\
& =E_{t, w}\left[W_{T}^{\pi^{*}}\right]-\frac{\gamma}{2 w} \operatorname{Var}_{t, w}\left[W_{T}^{\pi^{*}}\right] .
\end{aligned}
$$

We recall the probabilistic interpretations

$$
\begin{aligned}
f\left(t, w, y ; W_{T}^{\pi^{*}}\right) & =E_{t, w}\left[W_{T}^{\pi^{*}}\right]-\frac{\gamma}{2 y} E_{t, w}\left[\left(W_{T}^{\pi^{*}}\right)^{2}\right], \\
g\left(t, w ; W_{T}^{\pi^{*}}\right) & =E_{t, w}\left[W_{T}^{\pi^{*}}\right] .
\end{aligned}
$$

From these, we have

$$
V(t, w)=f\left(t, w, w ; W_{T}^{\pi^{*}}\right)+\frac{\gamma}{2 w} g^{2}\left(t, w ; W_{T}^{\pi^{*}}\right)
$$

By Proposition 3, we get the following results after a large number of elementary calculations.

Proposition 13. With notations as above, the extended HJB system takes the form

$$
\begin{aligned}
& f_{t}+ \frac{\gamma}{w} g g_{t}+\sup _{\pi \in \Pi}\left\{\left(f_{w}+\frac{\gamma}{w} g g_{w}\right)\right. \\
& \cdot\left(\pi_{1} \Delta_{u}^{1}+\pi_{2} \Delta_{u}^{2}+W_{t} r\right)+\frac{1}{2}\left(f_{w w}+\frac{\gamma}{w} g g_{w w}\right) \\
&\left.\cdot\left(\pi_{1}^{2} \Delta_{d}^{1}+2 \pi_{1} \pi_{2} \Delta_{d}^{2}+\pi_{2}^{2} \Delta_{d}^{3}\right)\right\}=0,
\end{aligned}
$$

and the optimal solution is given by

$$
\begin{aligned}
\pi_{1}^{*} & =\frac{f_{w}+(\gamma / w) g g_{w}}{f_{w w}+(\gamma / w) g g_{w w}} \frac{\Delta_{u}^{2} \Delta_{d}^{2}-\Delta_{u}^{1} \Delta_{d}^{3}}{\Delta_{d}^{1} \Delta_{d}^{3}-\left(\Delta_{d}^{2}\right)^{2}} \\
& =\frac{f_{w}+(\gamma / w) g g_{w}}{f_{w w}+(\gamma / w) g g_{w w}} \Delta_{m}^{1}, \\
\pi_{2}^{*} & =\frac{f_{w}+(\gamma / w) g g_{w}}{f_{w w}+(\gamma / w) g g_{w w}} \frac{\Delta_{u}^{1} \Delta_{d}^{2}-\Delta_{u}^{2} \Delta_{d}^{3}-\left(\Delta_{d}^{2}\right)^{2}}{f_{w}+(\gamma / w) g g_{w}} \Delta_{m}^{2} . \\
& =\frac{f_{w w}+(\gamma / w) g g_{w w}}{f_{w}}
\end{aligned}
$$

The proof is given in Appendix D.

Following Y. Li and Z. Li [21], we have two theorems as follows.

Theorem 14. The equilibrium time-consistent strategy for CIIP is given by

$$
\begin{aligned}
& \pi_{1}^{*}=c_{1}(t) w+k_{1}(t), \\
& \pi_{2}^{*}=c_{2}(t) w+k_{2}(t),
\end{aligned}
$$

where $c_{1}(t), c_{2}(t), k_{1}(t)$, and $k_{2}(t)$ satisfy the following integral equations:

$c_{1}(t)$

$$
=\Delta_{m}^{1}
$$

$\cdot\left(\frac{1}{\gamma} e^{\int_{t}^{T}\left[\Psi_{1}(s)-\Phi_{1}(s)\right] d s}+e^{\int_{t}^{T}\left[2 \Psi_{1}(s)-\Phi_{1}(s)\right] d s}-1\right)$,

$c_{2}(t)$

$=\Delta_{m}^{1}$

$\cdot\left(\frac{1}{\gamma} e^{\int_{t}^{T}\left[\Psi_{1}(s)-\Phi_{1}(s)\right] d s}+e^{\int_{t}^{T}\left[2 \Psi_{1}(s)-\Phi_{1}(s)\right] d s}-1\right)$,

$k_{1}(t)$

$$
\begin{aligned}
= & -\Delta_{m}^{2} \\
& \cdot\left(\frac{1}{2}-e^{2 \int_{t}^{T} \Psi_{1}(s) d s}\right) \int_{t}^{T} e^{-\int_{t}^{s} \Phi_{1}(\tau) d \tau} \Phi_{2}(s) d s,
\end{aligned}
$$

$k_{2}(t)$

$$
\begin{aligned}
= & -\Delta_{m}^{1} \\
& \cdot\left(\frac{1}{2}-e^{2 \int_{t}^{T} \Psi_{1}(s) d s}\right) \int_{t}^{T} e^{-\int_{t}^{s} \Phi_{1}(\tau) d \tau} \Phi_{2}(s) d s,
\end{aligned}
$$

with

$$
\begin{aligned}
& \Psi_{1}(t)=c_{1}(t) \Delta_{u}^{1}+c_{2}(t) \Delta_{u}^{2}+r \\
& \Psi_{2}(t)=k_{1}(t) \Delta_{u}^{1}+k_{2}(t) \Delta_{u}^{2}, \\
& \Phi_{1}(t)=2\left(c_{1}(t) \Delta_{u}^{1}+c_{2}(t) \Delta_{u}^{2}+r\right)+c_{1}^{2}(t)(\beta+1)^{2} \\
& \cdot \sigma^{2}+c_{2}^{2}(t)\left(\left(\beta^{*}+1\right)^{2}\left(\sigma^{*}\right)^{2}+\sigma_{e}^{2}\right)+2 c_{1}(t) c_{2}(t) \\
& \cdot\left((\beta+1)\left(\beta^{*}+1\right) \sigma \sigma^{*} \rho_{12}+(\beta+1) \sigma \sigma_{e} \rho_{13}\right) \\
& +2 c_{2}^{2}(t)\left(\beta^{*}+1\right) \sigma^{*} \sigma_{e} \rho_{23}, \\
& \Phi_{2}(t)=2\left(k_{1}(t) \Delta_{u}^{1}+k_{2}(t) \Delta_{u}^{2}\right)+2 c_{1}(t) k_{1}(t) \\
& \cdot(\beta+1)^{2} \sigma^{2}+2 c_{2}(t) k_{2}(t)\left(\left(\beta^{*}+1\right)^{2}\left(\sigma^{*}\right)^{2}+\sigma_{e}^{2}\right) \\
& +2\left(c_{1}(t) k_{2}(t)+c_{2}(t) k_{1}(t)\right) \\
& \cdot\left((\beta+1)\left(\beta^{*}+1\right) \sigma \sigma^{*} \rho_{12}+(\beta+1) \sigma \sigma_{e} \rho_{13}\right) \\
& +4 c_{2}(t) k_{2}(t)\left(\beta^{*}+1\right) \sigma^{*} \sigma_{e} \rho_{23}, \\
& +\Phi_{2}^{2}(t)\left(\beta^{*}+1\right) \sigma^{*} \sigma_{e} \rho_{23}, \\
& +\left(\left(\beta^{*}+1\right)^{2}\left(\sigma^{*}\right)^{2}+\sigma_{e}^{2}\right)+k_{1}(t) k_{2}(t) \\
& \left.\cdot(\beta+1)\left(\beta^{*}+1\right) \sigma \sigma^{*} \rho_{12}+(\beta+1) \sigma \sigma_{e} \rho_{13}\right) \\
& +\sigma^{2}+k_{2}^{2}(t)
\end{aligned}
$$

and the symbols $\Delta_{u}^{1}, \Delta_{u}^{2}$ are given in (45).

The proof is given in Appendix E. 


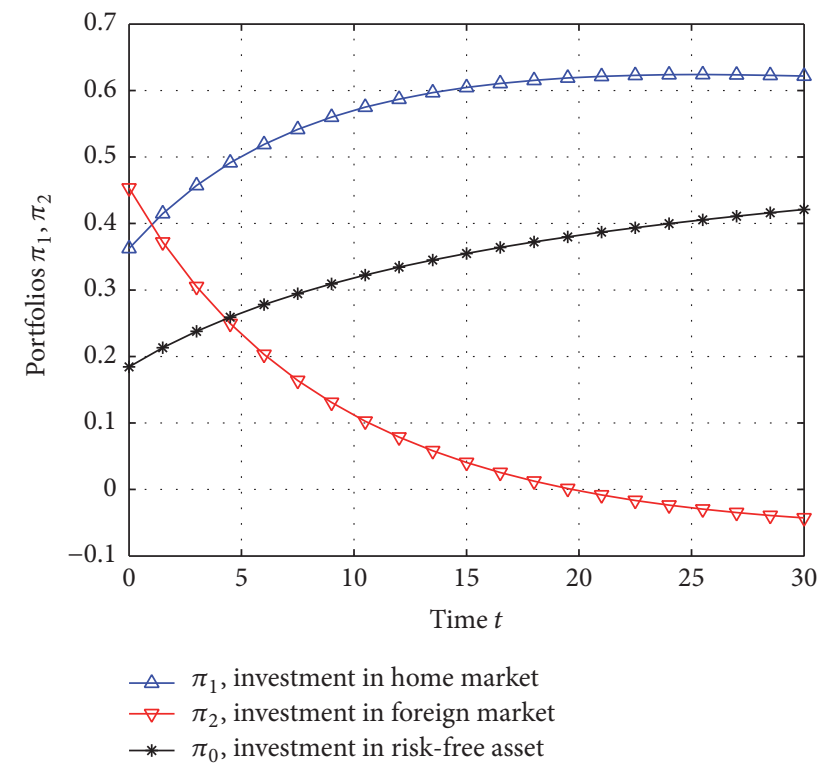

FIgure 1: Portfolio changes with time $t$.

Theorem 15. Integral equations (65) admit a unique solution $c_{1}(t), c_{2}(t), k_{1}(t), k_{2}(t) \in C^{1}[0, T] \cup C^{2}[0, T]$.

The proof can be seen in Y. Li and Z. Li [21].

\section{Simulating Results}

In this section, we give some simulating results of the optimal investment strategy for CIIP. Suppose that the time unit in the model is one year and let $\beta=-0.02, \beta^{*}=-0.02, \mu_{p}=$ $0.08, \mu_{p^{*}}=0.06, \mu_{c}=0.02, \mu_{c^{*}}=0.03, \sigma=0.2, \sigma^{*}=0.15$, $\sigma_{e}=0.05, \rho_{12}=0.8, \rho_{13}=0.6, \rho_{23}=0.4, \mu_{e}=0.02, \lambda_{R}=$ $0.005, \lambda_{R^{*}}=0.01, \lambda_{e}=0.01, P_{0}=6=P_{0}^{*}$, and $C_{0}=2=$ $C_{0}^{*}$. Besides, let the interest rate $r=0.003$, and risk aversion coefficient $\gamma=5$. For simplifying, we set $\pi_{1}=\pi_{1}^{*} / W_{t}, \pi_{2}=$ $\pi_{2}^{*} / W_{t}$, and then $\pi_{0}=1-\pi_{1}^{*} / W_{t}-\pi_{2}^{*} / W_{t}$.

Example 16. In this example, we present that the optimal investment strategy $\pi=\left(\pi_{1}, \pi_{2}\right)$ changes with time $t$.

As can be seen from Figure 1 , the variable $\pi_{1}$ increases with respect to time $t$, which shows that the money invested in home market is growing over time. The variable $\pi_{2}$ decreases with respect to time $t$, which shows that the money invested in foreign market is declining over time. And the trend of variable $\pi_{0}=1-\pi_{1}-\pi_{2}$, representing the money invested in risk-free asset, is similar to $\pi_{1}$.

Example 17. In this example, we present that the optimal investment strategy $\pi=\left(\pi_{1}, \pi_{2}\right)$ changes with parameters $\lambda_{R^{*}}, \lambda_{R}, \lambda_{e}$, and $\mu_{e}$.

To find the relationship between the portfolio and a single parameter, we set other parameters equal to zero. Besides, let $T=30$ and $t=5$, so we get four figures for different parameters.
In Figure 2(a), we get the curve demonstrating that the portfolio changes with home market information cost $\lambda_{R}$. When $\lambda_{R}=-0.035$, we can get the optimal investment strategy $\pi_{1}=0.2671, \pi_{2}=0.1714$, and $\pi_{0}=0.5615$, which shows that $26.71 \%$ of the wealth should be invested in home market, $17.14 \%$ of the wealth should be invested in foreign market, and $56.15 \%$ of that should be invested in risk-free asset.

In Figure 2(b), we get the curve demonstrating that the portfolio changes with foreign market information cost $\lambda_{R^{*}}$. When $\lambda_{R^{*}}=0.03$, we can get the optimal investment strategy $\pi_{1}=0.1899, \pi_{2}=0.4975$, and $\pi_{0}=0.3127$, which shows that $18.99 \%$ of the wealth should be invested in home market, $49.75 \%$ of the wealth should be invested in foreign market, and $31.27 \%$ of that should be invested in risk-free asset.

In Figure 2(c), we get the curve demonstrating that the portfolio changes with exchange market information cost $\lambda_{e}$. When $\lambda_{e}=0.045$, we can get the optimal investment strategy $\pi_{1}=0.4976, \pi_{2}=0.2006$, and $\pi_{0}=0.3018$, which shows that $49.76 \%$ of the wealth should be invested in home market, $20.06 \%$ of the wealth should be invested in foreign market, and $30.18 \%$ of that should be invested in risk-free asset.

In Figure 2(d), we get the curve demonstrating that the portfolio changes with exchange rate $\mu_{e}$. When $\mu_{e}=0.025$, we can get the optimal investment strategy $\pi_{1}=0.0873$, $\pi_{2}=0.5964$, and $\pi_{0}=0.3163$, which shows that $8.73 \%$ of the wealth should be invested in home market, $59.64 \%$ of the wealth should be invested in foreign market, and $31.63 \%$ of that should be invested in risk-free asset.

\section{Conclusion}

In this paper, we analyze a continuous-time model for corporate international investment problem (CIIP) with meanvariance criterion. Inspired by Björk and Murgoci [7], based on Nash subgame perfect equilibrium theory, we define an infinitesimal operator and deduce an extended HJB equation in continuous time. Besides, combining Björk and Murgoci [7] with Bellalah and $\mathrm{Wu}$ [33], we obtain the equilibrium time-consistent strategy for corporate international investment problem. In addition, following Björk et al. [37] and Y. Li and $\mathrm{Z}$. Li [21], we discuss two cases of risk aversion coefficient: one is constant and the other is state dependent. Finally, the simulating results are given to illustrate our conclusion and the influence of some parameters on the optimal solution.

Several possible extensions of this study may be interesting. Firstly, our study could be extended to stochastic interest rate and inflation. Secondly, jump-diffusion could be taken into account in our model. Thirdly, we could also consider that the prices of input and output hold for CEV model or mean-reverse model.

\section{Appendix}

\section{A. Deriving the Dynamics Price Process}

Generally, we consider the dynamics output price process

$$
d P_{t}=P_{t}\left(\mu_{p} d t+\sigma d B_{t}^{1}\right) .
$$




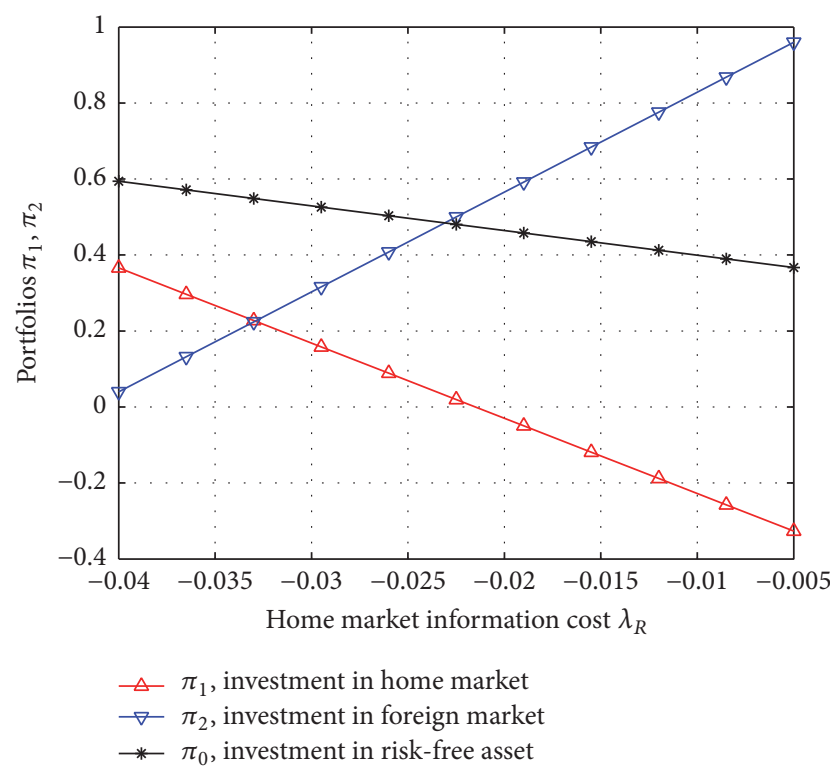

(a)

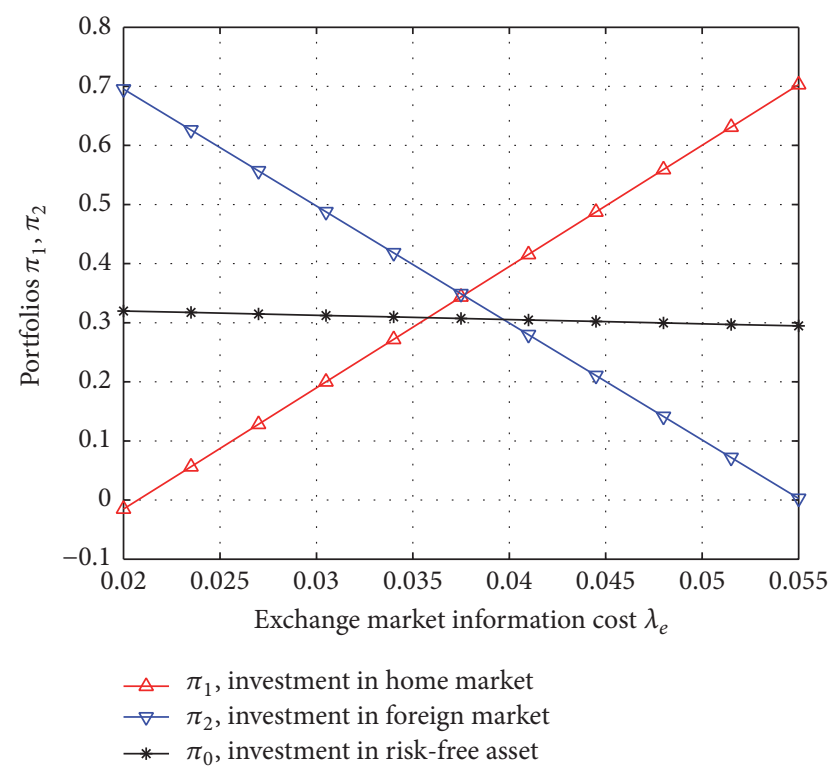

(c)

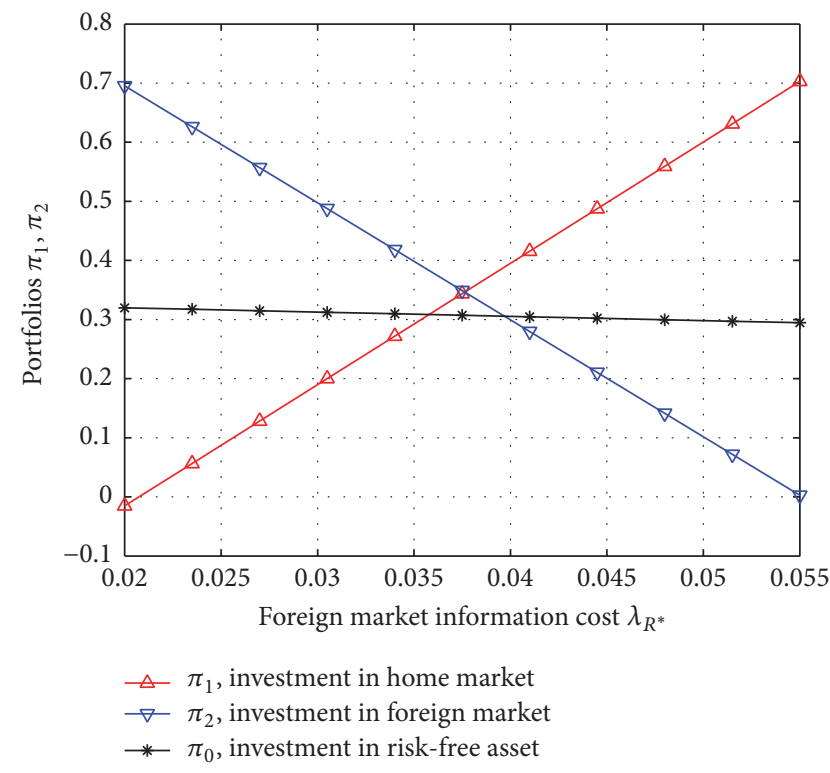

(b)

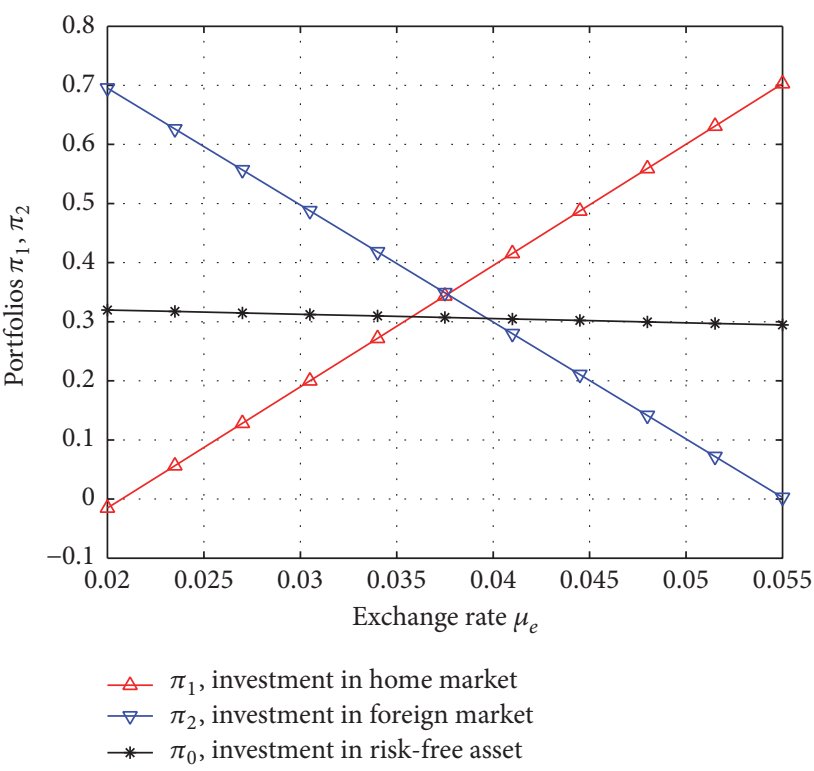

(d)

Figure 2: (a) Portfolio changes with home market information cost $\lambda_{R}$. (b) Portfolio changes with foreign market information cost $\lambda_{R^{*}}$. (c) Portfolio changes with exchange market information cost $\lambda_{e}$. (d) Portfolio changes with exchange rate $\mu_{e}$.

Denote that $Y=\ln P$, which means that $Y_{t}=0, Y_{P}=1 / P$, and $Y_{P P}=-1 / P^{2}$. By Itô Lemma, we obtain

$$
\begin{aligned}
d Y & =d \ln P_{t}=Y_{t} d t+Y_{P} d P+\frac{1}{2} Y_{P P}(d P)^{2} \\
& =\left(\mu_{p}-\frac{1}{2} \sigma^{2}\right) d t+\sigma d B_{t}^{1} .
\end{aligned}
$$

Therefore, we obtain

$$
P_{t}=P_{0} e^{\left(\mu_{p}-(1 / 2) \sigma^{2}\right) t} e^{\sigma B_{t}^{1}}
$$

Similarly, we have

$$
\begin{aligned}
P_{t}^{*} & =P_{0}^{*} e^{\left[\mu_{p^{*}}-(1 / 2)\left(\sigma^{*}\right)^{2}\right] t} e^{\sigma B_{t}^{2}}, \\
C_{t} & =C_{0} e^{\left(\mu_{c}-(1 / 2) \sigma^{2}\right) t} e^{\sigma B_{t}^{1}}, \\
C_{t}^{*} & =C_{0}^{*} e^{\left[\mu_{c^{*}}-(1 / 2)\left(\sigma^{*}\right)^{2}\right] t} e^{\sigma B_{t}^{2}}, \\
e_{t} & =e_{0} e^{\left(\mu_{e}-(1 / 2) \sigma_{e}^{2}\right) t} e^{\sigma B_{t}^{3}} .
\end{aligned}
$$

Now, we derive the dynamics cash flow process in home market. Notice that

$$
R_{t}=(1-\tau) P_{t}^{\beta}\left(P_{t}-C_{t}\right) .
$$


Denote that $\widetilde{Y}=(1-\tau) P^{\beta}(P-C)=(1-\tau) P^{\beta+1}-(1-\tau) P^{\beta} C$, which means that

$$
\begin{aligned}
\tilde{Y}_{t}= & 0, \\
\tilde{Y}_{P}= & (1-\tau)(\beta+1) P^{\beta}-(1-\tau) \beta P^{\beta-1} C, \\
\widetilde{Y}_{P P}= & (1-\tau) \beta(\beta+1) P^{\beta-1} \\
& -(1-\tau) \beta(\beta-1) P^{\beta-2} C, \\
\widetilde{Y}_{C}= & -(1-\tau) P^{\beta}, \\
\tilde{Y}_{C C}= & 0, \\
\tilde{Y}_{P C}= & -(1-\tau) \beta P^{\beta-1} .
\end{aligned}
$$

Similar to (A.2), we get

$$
\begin{aligned}
d \widetilde{Y} & =d R_{t}=\widetilde{Y}_{t} d t+\widetilde{Y}_{P} d P+\frac{1}{2} \widetilde{Y}_{P P}(d P)^{2}+\widetilde{Y}_{C} d C \\
& +\frac{1}{2} \widetilde{Y}_{C C}(d C)^{2}+\widetilde{Y}_{P C} d P d C=(1-\tau) P^{\beta}(P-C) \\
& .\left\{\left[(\beta+1)\left(\mu_{p}+\frac{1}{2} \beta \sigma^{2}\right)+\frac{\left(\mu_{p}-\mu_{c}\right) C}{P-C}\right] d t\right. \\
& \left.+(\beta+1) \sigma d B_{t}^{1}\right\} .
\end{aligned}
$$

Notice that the term $\left(\mu_{p}-\mu_{c}\right) C /(P-C)$ existed in the above equation. By (A.1)-(A.3), we have

$$
\begin{aligned}
\frac{C}{P-C} & =\frac{C_{0} e^{\left(\mu_{c}-(1 / 2) \sigma^{2}\right) t} e^{\sigma B_{t}^{1}}}{P_{0} e^{\left(\mu_{p}-(1 / 2) \sigma^{2}\right) t} e^{\sigma B_{t}^{1}}-C_{0} e^{\left(\mu_{c}-(1 / 2) \sigma^{2}\right) t} e^{\sigma B_{t}^{1}}} \\
& =\frac{C_{0} e^{\mu_{c} t}}{P_{0} e^{\mu_{p} t}-C_{0} e^{\mu_{c}} t}
\end{aligned}
$$

and the fact

$$
\begin{aligned}
(\beta & +1)\left(\mu_{p}+\frac{1}{2} \beta \sigma^{2}\right) \\
& =\frac{1}{2}(\beta+1)^{2} \sigma^{2}+(\beta+1)\left(\mu_{p}-\frac{1}{2} \sigma^{2}\right) .
\end{aligned}
$$

The dynamics cash flow process in home market can be expressed as

$$
\frac{d R_{t}}{R_{t}}=\widehat{f}(t) d t+(\beta+1) \sigma d B_{t}^{1},
$$

where

$$
\begin{aligned}
\widehat{f}(t)= & \frac{1}{2}(\beta+1)^{2} \sigma^{2}+(\beta+1)\left(\mu_{p}-\frac{1}{2} \sigma^{2}\right) \\
& +\frac{\left(\mu_{p}-\mu_{c}\right) C_{0} e^{\mu_{c} t}}{P_{0} e^{\mu_{p} t}-C_{0} e^{\mu_{c} t}} .
\end{aligned}
$$

Similarly, we can derive the dynamics cash flow process in foreign market before exchanging as follows:

$$
\frac{d \bar{R}_{t}}{\bar{R}_{t}}=\bar{f}(t) d t+\left(\beta^{*}+1\right) \sigma^{*} d B_{t}^{2},
$$

where

$$
\begin{aligned}
\bar{f}(t)= & \frac{1}{2}\left(\beta^{*}+1\right)^{2}\left(\sigma^{*}\right)^{2} \\
& +\left(\beta^{*}+1\right)\left(\mu_{p^{*}}-\frac{1}{2}\left(\sigma^{*}\right)^{2}\right) \\
& +\frac{\left(\mu_{p^{*}}-\mu_{c^{*}}\right) C_{0}^{*} e^{\mu_{c}^{*} t}}{P_{0}^{*} e^{\mu_{p^{*}} t}-C_{0}^{*} e^{\mu_{c^{*}} t}} .
\end{aligned}
$$

Then, by $R^{*}=e_{t} \bar{R}_{t}$, the dynamics cash flow process in foreign market after exchanging is given by

$$
\begin{aligned}
d R^{*} & =d\left(e_{t} \bar{R}_{t}\right)=d\left(e_{t}\right) \bar{R}_{t}+e_{t} d\left(\bar{R}_{t}\right) \\
& +d\left(e_{t}\right) d\left(\bar{R}_{t}\right) \\
& =e_{t} \bar{R}_{t}\left\{\left[\bar{f}(t)+\left(\beta^{*}+1\right) \sigma^{*} \sigma_{e} \rho_{23}+\mu_{e}+\lambda_{e}\right] d t\right. \\
& \left.+\left(\beta^{*}+1\right) \sigma^{*} d B_{t}^{2}+\sigma_{e} d B_{t}^{3}\right\} .
\end{aligned}
$$

Therefore, we obtain the dynamics cash flow process coming from foreign market as follows:

$$
\begin{aligned}
\frac{d R^{*}}{R^{*}}= & {\left[\widehat{f}^{*}(t)+\mu_{e}+\lambda_{e}\right] d t+\left(\beta^{*}+1\right) \sigma^{*} d B_{t}^{2} } \\
& +\sigma_{e} d B_{t}^{3},
\end{aligned}
$$

where

$$
\widehat{f}^{*}(t)=\bar{f}(t)+\left(\beta^{*}+1\right) \sigma^{*} \sigma_{e} \rho_{23} .
$$

\section{B. Proof of Theorem 8}

We conjecture that the value functions $V(t, w)$ and $g(t, w$; $W_{T}^{\widehat{\pi}}$ ) have the following forms:

$$
\begin{aligned}
V(t, w) & =A(t) w+B(t), \\
A(T) & =1, \\
B(T) & =0, \\
g\left(t, w ; W_{T}^{\widehat{\pi}}\right) & =a(t) w+b(t), \\
a(T) & =1, \\
b(T) & =0 .
\end{aligned}
$$

$V(T, W(T))$ and $g\left(T, W(T) ; W_{T}^{\widehat{\pi}}\right)$ satisfy the boundary conditions $V(T, W(T))=W_{T}$ and $g\left(T, W(T) ; W_{T}^{\hat{\pi}}\right)=W_{T}$, 
respectively. Given the linear structure of dynamics as well as the boundary conditions, it is natural to make the ansatz

$$
\begin{aligned}
V_{t} & =\dot{A} w+\dot{B}, \\
g_{t} & =\dot{a} w+\dot{b}, \\
V_{w} & =A, \\
g_{w} & =a, \\
V_{w w} & =0, \\
g_{w w} & =0 .
\end{aligned}
$$

For convenience, we denote that

$$
\begin{aligned}
\Delta_{u}^{1} & =\widehat{f}^{*}(t)-r+\lambda_{R^{*}}+\mu_{e}+\lambda_{e}, \\
\Delta_{u}^{2} & =\widehat{f}(t)-r+\lambda_{R}, \\
\Delta_{d}^{1} & =(\beta+1)^{2} \sigma^{2}, \\
\Delta_{d}^{2} & =(\beta+1)\left(\beta^{*}+1\right) \sigma \sigma^{*}+(\beta+1) \sigma \sigma_{e}, \\
\Delta_{d}^{3} & =\left(\beta^{*}+1\right)^{2}\left(\sigma^{*}\right)^{2}+\sigma_{e}^{2}+2\left(\beta^{*}+1\right) \sigma^{*} \sigma_{e}, \\
\Delta_{m}^{1} & =\frac{\Delta_{u}^{1} \Delta_{d}^{3}-\Delta_{u}^{2} \Delta_{d}^{2}}{\Delta_{d}^{1} \Delta_{d}^{3}-\left(\Delta_{d}^{2}\right)^{2}}, \\
\Delta_{m}^{2} & =\frac{\Delta_{u}^{2} \Delta_{d}^{1}-\Delta_{u}^{1} \Delta_{d}^{2}}{\Delta_{d}^{1} \Delta_{d}^{3}-\left(\Delta_{d}^{2}\right)^{2}} .
\end{aligned}
$$

Then, by (38), the corporate wealth process can be written as

$$
\begin{aligned}
d W_{t}= & \left(\pi_{1} \Delta_{u}^{1}+\pi_{2} \Delta_{u}^{2}+W_{t} r\right) d t+\pi_{1}(\beta+1) \sigma d B_{t}^{1} \\
& +\pi_{2}\left(\beta^{*}+1\right) \sigma^{*} d B_{t}^{2}+\pi_{2} \sigma_{e} d B_{t}^{3}
\end{aligned}
$$

By (B.3) and (B.4), we have

$$
\left[d W_{t}\right]^{2}=\left(\pi_{1}^{2} \Delta_{d}^{1}+2 \pi_{1} \pi_{2} \Delta_{d}^{2}+\pi_{2}^{2} \Delta_{d}^{3}\right) d t
$$

By (B.4)-(B.5) and Proposition 6, we can deduce an extended HJB equation

$$
\begin{aligned}
0= & \sup _{\pi \in \Pi}\left\{A^{\pi} V(t, w)-\frac{1}{2}\left(\pi_{1}^{2} \Delta_{d}^{1}+2 \pi_{1} \pi_{2} \Delta_{d}^{1}\right.\right. \\
& \left.\left.+\pi_{1}^{2} \Delta_{d}^{1}\right) G^{\prime \prime} g_{w}^{2}\right\}=\sup _{\pi \in \Pi}\left\{V_{t}\right. \\
& +V_{w}\left(\pi_{1} \Delta_{u}^{1}+\pi_{2} \Delta_{u}^{2}+w r\right) \\
& \left.+\frac{1}{2}\left(V_{w w}-G^{\prime \prime} g_{w}^{2}\right)\left(\pi_{1}^{2} \Delta_{d}^{1}+2 \pi_{1} \pi_{2} \Delta_{d}^{2}+\pi_{2}^{2} \Delta_{d}^{3}\right)\right\}
\end{aligned}
$$

$$
\begin{aligned}
& =\sup _{\pi \in \Pi}\left\{V_{t}+V_{w} w r+\pi_{1} V_{w} \Delta_{u}^{1}+\pi_{2} V_{w} \Delta_{u}^{2}\right. \\
& +\frac{1}{2} \pi_{1}^{2}\left[V_{w w}-G^{\prime \prime} g_{w}^{2}\right] \Delta_{d}^{1} \\
& +\pi_{1} \pi_{2}\left(V_{w w}-G^{\prime \prime} g_{w}^{2}\right) \Delta_{d}^{2} \\
& \left.+\frac{1}{2} \pi_{2}^{2}\left(V_{w w}-G^{\prime \prime} g_{w}^{2}\right) \Delta_{d}^{3}\right\}
\end{aligned}
$$

Therefore, the HJB system can be expressed as

$$
\begin{aligned}
& \sup _{\pi \in \mathcal{U}}\left\{V_{t}+V_{w} w r+\pi_{1} V_{w} \Delta_{u}^{1}+\pi_{2} V_{w} \Delta_{u}^{2}\right. \\
& +\frac{1}{2} \pi_{1}^{2}\left[V_{w w}-G^{\prime \prime} g_{w}^{2}\right] \Delta_{d}^{1} \\
& +\pi_{1} \pi_{2}\left[V_{w w}-G^{\prime \prime} g_{w}^{2}\right] \Delta_{d}^{2} \\
& \left.\quad+\frac{1}{2} \pi_{2}^{2}\left[V_{w w}-G^{\prime \prime} g_{w}^{2}\right] \Delta_{d}^{3}\right\}=0 \\
& A^{\hat{\pi}} g\left(t, w ; W_{T}^{\hat{\pi}}\right)=0 .
\end{aligned}
$$

By the first-order conditions of (B.7), we have

$$
\begin{aligned}
& V_{w} \Delta_{u}^{1}+\left(V_{w w}-G^{\prime \prime} g_{w}^{2}\right)\left(\pi_{1} \Delta_{d}^{1}+\pi_{2} \Delta_{d}^{2}\right)=0, \\
& V_{w} \Delta_{u}^{2}+\left(V_{w w}-G^{\prime \prime} g_{w}^{2}\right)\left(\pi_{1} \Delta_{d}^{2}+\pi_{2} \Delta_{d}^{3}\right)=0,
\end{aligned}
$$

which implies that

$$
\begin{aligned}
\pi_{1}^{*} & =-\frac{V_{w}}{V_{w w}-G^{\prime \prime} g_{w}^{2}} \cdot \frac{\Delta_{u}^{1} \Delta_{d}^{3}-\Delta_{u}^{2} \Delta_{d}^{2}}{\Delta_{d}^{1} \Delta_{d}^{3}-\left(\Delta_{d}^{2}\right)^{2}} \\
& :=-\frac{V_{w}}{V_{w w}-G^{\prime \prime} g_{w}^{2}} \cdot \Delta_{m}^{1}, \\
\pi_{2}^{*} & =-\frac{V_{w}}{V_{w w}-G^{\prime \prime} g_{w}^{2}} \cdot \frac{\Delta_{u}^{2} \Delta_{d}^{1}-\Delta_{u}^{1} \Delta_{d}^{2}}{\Delta_{d}^{1} \Delta_{d}^{3}-\left(\Delta_{d}^{2}\right)^{2}} \\
& :=-\frac{V_{w}}{V_{w w}-G^{\prime \prime} g_{w}^{2}} \cdot \Delta_{m}^{2} .
\end{aligned}
$$

By (B.2), we have

$$
\frac{V_{w}}{V_{w w}-G^{\prime \prime} g_{w}^{2}}=-\frac{A}{\gamma a^{2}} .
$$

Therefore, the optimal strategy can be written as

$$
\begin{aligned}
& \pi_{1}^{*}=\frac{1}{\gamma} \frac{A}{a^{2}} \Delta_{m}^{1}, \\
& \pi_{2}^{*}=\frac{1}{\gamma} \frac{A}{a^{2}} \Delta_{m}^{2} .
\end{aligned}
$$


Incorporating (B.2) and (B.12) into (B.7), we have

$$
\begin{aligned}
0= & \sup _{\pi \in \Pi}\left\{V_{t}+V_{w} w r+\pi_{1} V_{w} \Delta_{u}^{1}+\pi_{2} V_{w} \Delta_{u}^{2}\right. \\
& +\frac{1}{2} \pi_{1}^{2}\left[V_{w w}-G^{\prime \prime} g_{w}^{2}\right] \Delta_{d}^{1} \\
& +\pi_{1} \pi_{2}\left[V_{w w}-G^{\prime \prime} g_{w}^{2}\right] \Delta_{d}^{2} \\
& \left.+\frac{1}{2} \pi_{2}^{2}\left[V_{w w}-G^{\prime \prime} g_{w}^{2}\right] \Delta_{d}^{3}\right\}=\dot{A} w+\dot{B}+A w r \\
& +\frac{1}{\gamma} \frac{A^{2}}{a^{2}} \Delta_{m}^{1} \Delta_{u}^{1}+\frac{1}{\gamma} \frac{A^{2}}{a^{2}} \Delta_{m}^{2} \Delta_{u}^{2}+\frac{1}{2}\left(\frac{1}{\gamma} \frac{A}{a^{2}} \Delta_{m}^{1}\right)^{2} \\
& \cdot\left[-\gamma a^{2}\right] \Delta_{d}^{1}+\frac{1}{\gamma} \frac{A}{a^{2}} \Delta_{m}^{1} \frac{1}{\gamma} \frac{A}{a^{2}} \Delta_{m}^{2}\left[-\gamma a^{2}\right] \Delta_{d}^{2} \\
& +\frac{1}{2}\left(\frac{1}{\gamma} \frac{A}{a^{2}} \Delta_{m}^{2}\right)^{2}\left[-\gamma a^{2}\right] \Delta_{d}^{3}=\dot{A} w+\dot{B}+A w r \\
& \left.-\left(\frac{1}{2}\left(\Delta_{m}^{1}\right)^{2} \Delta_{d}^{1}+\Delta_{m}^{1} \Delta_{m}^{2} \Delta_{d}^{2}+\frac{1}{2}\left(\Delta_{m}^{2}\right)^{2} \Delta_{d}^{3}\right)\right] \\
& +\frac{1}{\gamma} \Delta_{m}^{1} \Delta_{u}^{1}\left(\frac{A}{a}\right)^{2}+\frac{1}{\gamma} \Delta_{m}^{2} \Delta_{u}^{2}\left(\frac{A}{a}\right)^{2}-\frac{1}{2 \gamma}\left(\Delta_{m}^{1}\right)^{2} \\
& \cdot \Delta_{d}^{3}\left(\frac{A}{a}\right)^{2}=\left(\frac{A}{a}\right)^{2}-\frac{1}{\gamma} \Delta_{m}^{1} \Delta_{m}^{2} \Delta_{d}^{2}\left(\frac{A}{a}\right)^{2}-\frac{1}{2 \gamma}\left(\Delta_{m}^{2}\right)^{2} \\
& \left.+\Delta_{u}^{2}\right)
\end{aligned}
$$

As (B.13) must be satisfied for every $w$, the variables $A$ and $B$ must satisfy the following ordinary differential equation (ODE):

$$
\begin{aligned}
\dot{A}+A r & =0, \\
\dot{B}+\frac{1}{\gamma}\left(\frac{A}{a}\right)^{2}(\Lambda-\Gamma) & =0,
\end{aligned}
$$

where

$$
\begin{aligned}
& \Lambda(t)=\Delta_{m}^{1} \Delta_{u}^{1}+\Delta_{m}^{2} \Delta_{u}^{2}, \\
& \Gamma(t)=\frac{1}{2}\left(\Delta_{m}^{1}\right)^{2} \Delta_{d}^{1}+\Delta_{m}^{1} \Delta_{m}^{2} \Delta_{d}^{2}+\frac{1}{2}\left(\Delta_{m}^{2}\right)^{2} \Delta_{d}^{3} .
\end{aligned}
$$

By (B.14) and the boundary condition $A(T)=1$, we have

$$
A(t)=e^{-r(T-t)} .
$$

As (B.15) contains an unknown function $a$, we must use (B.8) to determine it. Inserting (B.2), (B.12), and (B.17) into (B.8), we have

$$
0=(\dot{a}+a r) w+\dot{b}+\frac{1}{\gamma} \frac{A}{a}\left(\Delta_{m}^{1} \Delta_{u}^{1}+\Delta_{m}^{2} \Delta_{u}^{2}\right) .
$$

Similar to (B.14) and (B.15), the functions $a$ and $b$ must satisfy the following ODE:

$$
\begin{aligned}
\dot{a}+a r & =0, \\
\dot{b}+\frac{1}{\gamma} \frac{A}{a} \Lambda & =0 .
\end{aligned}
$$

By (B.19) and the boundary condition $a(T)=1$, we have

$$
a(t)=e^{-r(T-t)} .
$$

Notice that (B.17) and (B.21) lead to $A / a=1$. Equation (B.20) and $b(T)=0$ give us

$$
b(t)=\frac{1}{\gamma} \int_{t}^{T} \Lambda(s) d s
$$

Meanwhile, (B.15) and $B(T)=0$ give us

$$
B(t)=\frac{1}{\gamma} \int_{t}^{T}[\Lambda(s)-\Gamma(s)] d s .
$$

Therefore, we obtain the equilibrium time-consistent strategy as follows:

$$
\begin{aligned}
& \pi_{1}^{*}=\frac{1}{\gamma} e^{r(T-t)} \Delta_{m}^{1}, \\
& \pi_{2}^{*}=\frac{1}{\gamma} e^{r(T-t)} \Delta_{m}^{2} .
\end{aligned}
$$

And the value functions $V$ and $g$ are of the following forms:

$$
\begin{aligned}
& V(t, w)=e^{r(T-t)} w+\frac{1}{\gamma} \int_{t}^{T}[\Lambda(s)-\Gamma(s)] d s, \\
& g(t, w)=e^{r(T-t)} w+\frac{1}{\gamma} \int_{t}^{T} \Lambda(s) d s,
\end{aligned}
$$

where $\Lambda(s), \Gamma(s)$ are given in (B.16) and $\Delta_{u}^{1}, \Delta_{u}^{2}, \Delta_{d}^{1}, \Delta_{d}^{2}, \Delta_{d}^{3}$, $\Delta_{m}^{1}$, and $\Delta_{m}^{2}$ are given in (B.3).

Then, we complete the proof.

\section{Proof of Theorem 11}

According to the definition of value function

$$
\begin{aligned}
V(t, w) & =J\left(t, w, \pi^{*}\right) \\
& =E_{t, w}\left[W_{T}^{\pi^{*}}\right]-\frac{\gamma}{2} \operatorname{Var}_{t, w}\left[W_{T}^{\pi^{*}}\right]
\end{aligned}
$$

and the optimal value function given in Theorem 8 ,

$$
\begin{aligned}
V(t, w) & =e^{r(T-t)} w+\frac{1}{\gamma} \int_{t}^{T}[\Lambda(s)-\Gamma(s)] d s, \\
g\left(t, w ; W_{T}^{\pi^{*}}\right) & =e^{r(T-t)} w+\frac{1}{\gamma} \int_{t}^{T} \Lambda(s) d s .
\end{aligned}
$$


And noticing that $g\left(t, w ; W_{T}^{\pi^{*}}\right)=E_{t, w}\left[W_{T}^{\pi^{*}}\right]$, we have

$$
\begin{aligned}
\operatorname{Var}_{t, w}\left[W_{T}^{\pi^{*}}\right] & =\frac{2}{\gamma}\left(E_{t, w}\left[W_{T}^{\pi^{*}}\right]-V(t, w)\right) \\
& =\frac{2}{\gamma}\left(g\left(t, w ; W_{T}^{\pi^{*}}\right)-V(t, w)\right) \\
& =\frac{2}{\gamma^{2}} \int_{t}^{T} \Gamma(s) d s,
\end{aligned}
$$

which leads to

$$
\frac{1}{\gamma}=\sqrt{\frac{\operatorname{Var}_{t, w}\left[W_{T}^{\pi^{*}}\right]}{2 \int_{t}^{T} \Gamma(s) d s}} .
$$

Then, incorporating it into (C.3), we have

$$
\begin{aligned}
g\left(t, w ; W_{T}^{\pi^{*}}\right)= & e^{r(T-t)} w+\frac{1}{\gamma} \int_{t}^{T} \Lambda(s) d s \\
= & e^{r(T-t)} w+\sqrt{\frac{\operatorname{Var}_{t, w}\left[W_{T}^{\pi^{*}}\right]}{2 \int_{t}^{T} \Gamma(s) d s}} \\
& \cdot \int_{t}^{T} \Lambda(s) d s \\
= & e^{r(T-t)} w+\sqrt{\operatorname{Var}_{t, w}\left[W_{T}^{\pi^{*}}\right]} \\
& \cdot \frac{\int_{t}^{T} \Lambda(s) d s}{\sqrt{2 \int_{t}^{T} \Gamma(s) d s}} .
\end{aligned}
$$

Therefore, we have

$$
\begin{gathered}
E_{t, w}\left[W_{T}^{\pi^{*}}\right]=e^{r(T-t)} w-\sqrt{\operatorname{Var}_{t, w}\left[W_{T}^{\pi^{*}}\right]} \\
\cdot \frac{\int_{t}^{T} \Lambda(s) d s}{\sqrt{2 \int_{t}^{T} \Gamma(s) d s}} .
\end{gathered}
$$

Hereto, we complete the proof.

\section{Proof of Proposition 13}

Firstly, by (B.4) and (B.5), we have

$$
\begin{aligned}
& \left(A^{\pi} V\right)(t, w)=V_{t}+V_{w}\left(\pi_{1} \Delta_{u}^{1}+\pi_{2} \Delta_{u}^{2}+W_{t} r\right)+\frac{1}{2} \\
& \cdot V_{w w}\left(\pi_{1}^{2} \Delta_{d}^{1}+2 \pi_{1} \pi_{2} \Delta_{d}^{2}+\pi_{2}^{2} \Delta_{d}^{3}\right)
\end{aligned}
$$

$\left(A^{\pi} f\right)\left(t, w, w ; W_{T}^{\pi}\right)=f_{t}+\left(f_{w}+f_{v}\right)$

$$
\begin{aligned}
& \cdot\left(\pi_{1} \Delta_{u}^{1}+\pi_{2} \Delta_{u}^{2}+W_{t} r\right)+\frac{1}{2}\left(f_{w w}+2 f_{w v}+f_{v v}\right) \\
& \cdot\left(\pi_{1}^{2} \Delta_{d}^{1}+2 \pi_{1} \pi_{2} \Delta_{d}^{2}+\pi_{2}^{2} \Delta_{d}^{3}\right), \\
& \left(A^{\pi} f^{w}\right)\left(t, w ; W_{T}^{\pi}\right)=f_{t}+f_{w}\left(\pi_{1} \Delta_{u}^{1}+\pi_{2} \Delta_{u}^{2}+W_{t} r\right) \\
& +\frac{1}{2} f_{w w}\left(\pi_{1}^{2} \Delta_{d}^{1}+2 \pi_{1} \pi_{2} \Delta_{d}^{2}+\pi_{2}^{2} \Delta_{d}^{3}\right), \\
& \left(A^{\pi}(G \diamond g)\right)\left(t, w ; W_{T}^{\pi}\right)=G_{v} g_{t}+\left(G_{w}+G_{v} g_{w}\right) \\
& \cdot\left(\pi_{1} \Delta_{u}^{1}+\pi_{2} \Delta_{u}^{2}+W_{t} r\right) \\
& +\frac{1}{2}\left(G_{w w}+G_{v v} g_{w}^{2}+G_{v} g_{w w}+2 G_{w v} g_{w}\right) \\
& \cdot\left(\pi_{1}^{2} \Delta_{d}^{1}+2 \pi_{1} \pi_{2} \Delta_{d}^{2}+\pi_{2}^{2} \Delta_{d}^{3}\right),
\end{aligned}
$$

where $G=G\left(w, g\left(t, w ; W_{T}^{\pi}\right)\right), g=g\left(t, w ; W_{T}^{\pi}\right)$, and

$$
\begin{aligned}
& \left(H_{g}^{\pi} G\right)\left(t, w ; W_{T}^{\pi}\right)=G_{v}\left[g_{t}\right. \\
& \quad+g_{w}\left(\pi_{1} \Delta_{u}^{1}+\pi_{2} \Delta_{u}^{2}+W_{t} r\right) \\
& \left.\quad+\frac{1}{2} g_{w w}\left(\pi_{1}^{2} \Delta_{d}^{1}+2 \pi_{1} \pi_{2} \Delta_{d}^{2}+\pi_{2}^{2} \Delta_{d}^{3}\right)\right] .
\end{aligned}
$$

The extended HJB system is thus as follows:

$$
\begin{aligned}
V_{t} & +\sup _{\pi \in \Pi}\left\{\left(V_{w}-f_{v}-G_{w}\right)\left(\pi_{1} \Delta_{u}^{1}+\pi_{2} \Delta_{u}^{2}+W_{t} r\right)\right. \\
& +\frac{1}{2}\left[V_{w w}-2 f_{w v}-f_{v v}-G_{w w}-G_{v v} g_{w}^{2}\right. \\
& \left.\left.-2 G_{w v} g_{w}\right]\left(\pi_{1}^{2} \Delta_{d}^{1}+2 \pi_{1} \pi_{2} \Delta_{d}^{2}+\pi_{2}^{2} \Delta_{d}^{3}\right)\right\}=0
\end{aligned}
$$

$$
\begin{aligned}
f_{t} & +f_{w}\left(\pi_{1}^{*} \Delta_{u}^{1}+\pi_{2}^{*} \Delta_{u}^{2}+W_{t} r\right)+\frac{1}{2} f_{w w}\left(\left(\pi_{1}^{*}\right)^{2} \Delta_{d}^{1}\right. \\
& \left.+2 \pi_{1}^{*} \pi_{2}^{*} \Delta_{d}^{2}+\left(\pi_{2}^{*}\right)^{2} \Delta_{d}^{3}\right)=0, \\
g_{t} & +g_{w}\left(\pi_{1}^{*} \Delta_{u}^{1}+\pi_{2}^{*} \Delta_{u}^{2}+W_{t} r\right)+\frac{1}{2} g_{w w}\left(\left(\pi_{1}^{*}\right)^{2} \Delta_{d}^{1}\right. \\
& \left.+2 \pi_{1}^{*} \pi_{2}^{*} \Delta_{d}^{2}+\left(\pi_{2}^{*}\right)^{2} \Delta_{d}^{3}\right)=0 .
\end{aligned}
$$

Noticing that $G(w, v)=(\gamma / 2 w) v^{2}$ and $V(t, w)=f(t, w, v$; $\left.W_{T}^{\pi^{*}}\right)+(\gamma / 2 w) g^{2}\left(t, w ; W_{T}^{\pi^{*}}\right)$, we obtain

$$
\begin{aligned}
G_{w} & =-\frac{\gamma}{2 w^{2}} v^{2}, \\
G_{v} & =\frac{\gamma}{w} v, \\
G_{w w} & =\frac{\gamma}{w^{3}} v^{2},
\end{aligned}
$$




$$
\begin{aligned}
G_{w v}= & \frac{\gamma}{w^{2}} v, \\
G_{v v}= & \frac{\gamma}{w} \\
V_{t}= & f_{t}+\frac{\gamma}{w} g g_{t}, \\
V_{w}= & f_{w}+f_{v}-\frac{\gamma}{2 w^{2}} g^{2}+\frac{\gamma}{w} g g_{w}, \\
V_{w w}= & f_{w w}+2 f_{w v}+f_{v v}+\frac{\gamma}{w^{3}} g^{2}-\frac{2 \gamma}{w^{2}} g g_{w}+\frac{\gamma}{w} g_{w}^{2} \\
& +\frac{\gamma}{w} g g_{w w} .
\end{aligned}
$$

Using these expressions, the HJB equation becomes

$$
\begin{aligned}
& f_{t}+\frac{\gamma}{w} g g_{t}+\sup _{\pi \in \Pi}\left\{\left(f_{w}+\frac{\gamma}{w} g g_{w}\right)\right. \\
& \cdot\left(\pi_{1} \Delta_{u}^{1}+\pi_{2} \Delta_{u}^{2}+W_{t} r\right)+\frac{1}{2}\left(f_{w w}+\frac{\gamma}{w} g g_{w w}\right) \\
&\left.\cdot\left(\pi_{1}^{2} \Delta_{d}^{1}+2 \pi_{1} \pi_{2} \Delta_{d}^{2}+\pi_{2}^{2} \Delta_{d}^{3}\right)\right\}=0 .
\end{aligned}
$$

$$
\begin{aligned}
& \left(f_{w}+\frac{\gamma}{w} g g_{w}\right) \Delta_{u}^{2} \\
& +\left(f_{w w}+\frac{\gamma}{w} g g_{w w}\right)\left(\pi_{1} \Delta_{d}^{2}+\pi_{2} \Delta_{d}^{3}\right)=0 .
\end{aligned}
$$

Compared to (B.9) and (B.10), we obtain the optimal strategy as follows:

$$
\begin{aligned}
& \pi_{1}^{*}=\frac{f_{w}+(\gamma / w) g g_{w}}{f_{w w}+(\gamma / w) g g_{w w}} \Delta_{m}^{1}, \\
& \pi_{2}^{*}=\frac{f_{w}+(\gamma / w) g g_{w}}{f_{w w}+(\gamma / w) g g_{w w}} \Delta_{m}^{2} .
\end{aligned}
$$

Now, we complete the proof.

\section{E. Proof of Theorem 14}

Following Y. Li and Z. Li [21], we conjecture that $\pi_{1}^{*}$ and $\pi_{2}^{*}$ are affine form of $w$, so we guess that

$$
\begin{aligned}
& \pi_{1}^{*}=c_{1}(t) w+k_{1}(t), \\
& \pi_{2}^{*}=c_{2}(t) w+k_{2}(t),
\end{aligned}
$$

for some deterministic functions $c_{1}, k_{1}, c_{2}, k_{2}$. In this case, the wealth process $W$ is

$$
\begin{aligned}
d W= & {\left[\Psi_{1}(t) W+\Psi_{2}(t) r\right] d t } \\
& +\left[c_{1}(t) W+k_{1}(t)\right](\beta+1) \sigma d B_{t}^{1} \\
& +\left[c_{2}(t) W+k_{2}(t)\right]\left(\beta^{*}+1\right) \sigma^{*} d B_{t}^{2} \\
& +\left[c_{2}(t) W+k_{2}(t)\right] \sigma_{e} d B_{t}^{3},
\end{aligned}
$$

where

$$
\begin{aligned}
\Psi_{1}(t)= & c_{1}(t) \Delta_{u}^{1}+c_{2}(t) \Delta_{u}^{2}+r \\
\Psi_{2}(t)= & k_{1}(t) \Delta_{u}^{1}+k_{2}(t) \Delta_{u}^{2}, \\
d W^{2}= & {\left[\Phi_{1}(t) W^{2}+\Phi_{2}(t) W+\Phi_{3}(t)\right] d t } \\
& +2\left[c_{1}(t) W^{2}+k_{1}(t) W\right](\beta+1) \sigma d B_{t}^{1} \\
& +2\left[c_{2}(t) W^{2}+k_{2}(t) W\right]\left(\beta^{*}+1\right) \sigma^{*} d B_{t}^{2} \\
& +2\left[c_{2}(t) W^{2}+k_{2}(t) W\right] \sigma_{e} d B_{t}^{3},
\end{aligned}
$$

where

$$
\begin{aligned}
& \Phi_{1}(t)=2\left(c_{1}(t) \Delta_{u}^{1}+c_{2}(t) \Delta_{u}^{2}+r\right)+c_{1}^{2}(t)(\beta+1)^{2} \\
& \cdot \sigma^{2}+c_{2}^{2}(t)\left(\left(\beta^{*}+1\right)^{2}\left(\sigma^{*}\right)^{2}+\sigma_{e}^{2}\right)+2 c_{1}(t) c_{2}(t) \\
& \cdot\left((\beta+1)\left(\beta^{*}+1\right) \sigma \sigma^{*} \rho_{12}+(\beta+1) \sigma \sigma_{e} \rho_{13}\right) \\
& +2 c_{2}^{2}(t)\left(\beta^{*}+1\right) \sigma^{*} \sigma_{e} \rho_{23}, \\
& \Phi_{2}(t)=2\left(k_{1}(t) \Delta_{u}^{1}+k_{2}(t) \Delta_{u}^{2}\right)+2 c_{1}(t) k_{1}(t) \\
& \cdot(\beta+1)^{2} \sigma^{2}+2 c_{2}(t) k_{2}(t)\left(\left(\beta^{*}+1\right)^{2}\left(\sigma^{*}\right)^{2}+\sigma_{e}^{2}\right) \\
& +2\left(c_{1}(t) k_{2}(t)+c_{2}(t) k_{1}(t)\right) \\
& \cdot\left((\beta+1)\left(\beta^{*}+1\right) \sigma \sigma^{*} \rho_{12}+(\beta+1) \sigma \sigma_{e} \rho_{13}\right) \\
& +4 c_{2}(t) k_{2}(t)\left(\beta^{*}+1\right) \sigma^{*} \sigma_{e} \rho_{23}, \\
& \Phi_{3}(t)=k_{1}^{2}(t)(\beta+1)^{2} \sigma^{2}+k_{2}^{2}(t) \\
& \quad \cdot\left(\left(\beta^{*}+1\right)^{2}\left(\sigma^{*}\right)^{2}+\sigma_{e}^{2}\right)+k_{1}(t) k_{2}(t) \\
& +\left((\beta+1)\left(\beta^{*}+1\right) \sigma \sigma^{*} \rho_{12}+(\beta+1) \sigma \sigma_{e} \rho_{13}\right) \\
& +k_{2}^{2}(t)\left(\beta^{*}+1\right) \sigma^{*} \sigma_{e} \rho_{23} .
\end{aligned}
$$

Then, a direct computation shows that

$$
\begin{aligned}
E_{t, w}\left[W_{T}^{\pi^{*}}\right] & =a(t)[w+h(t)], \\
E_{t, w}\left[\left(W_{T}^{\pi^{*}}\right)^{2}\right] & =b(t)\left[w^{2}+l(t) w+d(t)\right],
\end{aligned}
$$


where

$$
\begin{aligned}
& a(t)=e^{\int_{t}^{T} \Psi_{1}(s) d s}, \\
& h(t)=e^{-\int_{t}^{s} \Phi_{1}(\tau) d \tau} \Phi_{2}(s) d s, \\
& b(t)=e^{\int_{t}^{T} \Phi_{1}(s) d s}, \\
& l(t)=\int_{t}^{T} e^{-\int_{t}^{s} \Phi_{1}(\tau) d \tau} \Phi_{2}(s) d s, \\
& d(t)=\int_{t}^{T} e^{-\int_{t}^{s} \Phi_{1}(\tau) d \tau} \Phi_{3}(s) d s .
\end{aligned}
$$

We recall that

$$
\begin{aligned}
f\left(t, w, v ; W_{T}^{\pi^{*}}\right) & =E_{t, w}\left[W_{T}^{\pi^{*}}\right]-\frac{\gamma}{2 v} E_{t, w}\left[\left(W_{T}^{\pi^{*}}\right)^{2}\right], \\
g\left(t, w ; W_{T}^{\pi^{*}}\right) & =E_{t, w}\left[W_{T}^{\pi^{*}}\right] .
\end{aligned}
$$

So, we have

$$
\begin{aligned}
f\left(t, w, v ; W_{T}^{\pi^{*}}\right)= & a(t)[w+h(t)] \\
& -\frac{\gamma}{2 v} b(t)\left[w^{2}+l(t) w+d(t)\right], \\
g\left(t, w ; W_{T}^{\pi^{*}}\right)= & a(t)[w+h(t)], \\
f_{t}= & \dot{a}(t)[w+h(t)]+a(t) \dot{h}(t) \\
& -\frac{\gamma}{2 v} \dot{b}(t)\left[w^{2}+l(t) w+d(t)\right] \\
& -\frac{\gamma}{2 v} b(t)[\dot{l}(t) w+\dot{d}(t)], \\
f_{w}= & a(t)-\frac{\gamma}{2 v} b(t)[2 w+l(t)], \\
f_{w w}= & \frac{\gamma}{v} b(t), \\
g_{t}= & \dot{a}(t)[w+h(t)]+a(t) \dot{h}(t), \\
g_{w}= & a(t), \\
g_{w w}= & 0,
\end{aligned}
$$

where $\dot{a}(t)=\partial a / \partial t$ and so forth.

Now, we calculate the term $\left(f_{w}+(\gamma / w) g g_{w}\right) /\left(f_{w w}+\right.$ $\left.(\gamma / w) g g_{w w}\right)$ which exists in (D.7):

$$
\begin{aligned}
\frac{f_{w}+(\gamma / w) g g_{w}}{f_{w w}+(\gamma / w) g g_{w w}}= & -\frac{(1 / \gamma) a(t)+a^{2}(t)-b(t)}{b(t)} w \\
& +\frac{(1 / 2) b(t) l(t)-a^{2}(t) l(t)}{b(t)} .
\end{aligned}
$$

Denote that

$$
\begin{aligned}
\tilde{c}(t) & =\frac{(1 / \gamma) a(t)+a^{2}(t)-b(t)}{b(t)}, \\
\tilde{k}(t) & =\frac{(1 / 2) b(t) l(t)-a^{2}(t) l(t)}{b(t)} .
\end{aligned}
$$

Then, by (E.6), we have

$$
\begin{aligned}
& \widetilde{c}(t)=\frac{1}{\gamma} e^{\int_{t}^{T}\left[\Psi_{1}(s)-\Phi_{1}(s)\right] d s}+e^{\int_{t}^{T}\left[2 \Psi_{1}(s)-\Phi_{1}(s)\right] d s}-1, \\
& \widetilde{k}(t)=\left(\frac{1}{2}-e^{2 \int_{t}^{T} \Psi_{1}(s) d s}\right) \int_{t}^{T} e^{-\int_{t}^{s} \Phi_{1}(\tau) d \tau} \Phi_{2}(s) d s .
\end{aligned}
$$

Therefore, (D.7) can be written as

$$
\begin{aligned}
& \pi_{1}^{*}=\Delta_{m}^{1}[\widetilde{c}(t) w-\widetilde{k}(t)], \\
& \pi_{2}^{*}=\Delta_{m}^{2}[\widetilde{c}(t) w-\widetilde{k}(t)] .
\end{aligned}
$$
tions:

Compared to (E.1), we get the following integral equa-

$$
\begin{aligned}
c_{1}(t) & \\
= & \Delta_{m}^{1} \\
& \cdot\left(\frac{1}{\gamma} e^{\int_{t}^{T}\left[\Psi_{1}(s)-\Phi_{1}(s)\right] d s}+e^{\int_{t}^{T}\left[2 \Psi_{1}(s)-\Phi_{1}(s)\right] d s}-1\right), \\
c_{2}(t) & \quad \Delta_{m}^{2} \\
& \cdot\left(\frac{1}{\gamma} e^{\int_{t}^{T}\left[\Psi_{1}(s)-\Phi_{1}(s)\right] d s}+e^{\int_{t}^{T}\left[2 \Psi_{1}(s)-\Phi_{1}(s)\right] d s}-1\right), \\
k_{1}(t) & -\Delta_{m}^{1} \\
& \cdot\left(\frac{1}{2}-e^{2 \int_{t}^{T} \Psi_{1}(s) d s}\right) \int_{t}^{T} e^{-\int_{t}^{s} \Phi_{1}(\tau) d \tau} \Phi_{2}(s) d s, \\
k_{2}(t) & \cdot\left(\frac{1}{2}-e^{2 \int_{t}^{T} \Psi_{1}(s) d s}\right) \int_{t}^{T} e^{-\int_{t}^{s} \Phi_{1}(\tau) d \tau} \Phi_{2}(s) d s . \\
= & \Delta_{m}^{2} \\
= &
\end{aligned}
$$

Hereto, we complete the proof.

\section{Competing Interests}

The authors declare that there are no competing interests regarding the publication of this article.

\section{Acknowledgments}

This research is supported by grants from the National Natural Science Foundation of China (no. 71231008). 


\section{References}

[1] R. H. Strotz, "Myopia and inconsistency in dynamic utility maximization," The Review of Economic Studies, vol. 23, no. 3, pp. 165-180, 1955.

[2] S. M. Goldman, "Consistent plans," The Review of Economic Studies, vol. 47, no. 3, pp. 533-537, 1980.

[3] P. Krusell and A. A. Smith Jr., "Consumption-savings decisions with quasi-geometric discounting," Econometrica, vol. 71, no. 1, pp. 365-375, 2003.

[4] B. Peleg and M. E. Yaari, "On the existence of a consistent course of action when tastes are changing," The Review of Economic Studies, vol. 40, no. 3, pp. 391-401, 1973.

[5] R. A. Pollak, "Consistent planning," The Review of Economic Studies, vol. 35, no. 2, pp. 201-208, 1968.

[6] N. Vieille and J. W. Weibull, "Multiple solutions under quasiexponential discounting," Economic Theory, vol. 39, no. 3, pp. 513-526, 2009.

[7] T. Björk and A. Murgoci, "A general theory of Markovian timeinconsistent stochastic control," Working Paper, Stockholm School of Economics, Stockholm, Sweden, 2009.

[8] Y. Hu, H. Jin, and X. Y. Zhou, "Time-inconsistent stochastic linear-quadratic control," SIAM Journal on Control and Optimization, vol. 50, no. 3, pp. 1548-1572, 2012.

[9] J. Yong, "A deterministic linear quadratic time-inconsistent optimal control problem," Mathematical Control and Related Fields, vol. 1, no. 1, pp. 83-118, 2011.

[10] J. Yong, "Time-inconsistent optimal control problems and the equilibrium HJB equation," Mathematical Control and Related Fields, vol. 2, no. 3, pp. 271-329, 2012.

[11] C. Czichowsky, "Time-consistent mean-variance portfolio selection in discrete and continuous time," Finance and Stochastics, vol. 17, no. 2, pp. 227-271, 2013.

[12] J. Yong, "Linear-quadratic optimal control problems for meanfield stochastic differential equations," SIAM Journal on Control and Optimization, vol. 51, no. 4, pp. 2809-2838, 2013.

[13] T. Björk and A. Murgoci, "A theory of Markovian timeinconsistent stochastic control in discrete time," Finance and Stochastics, vol. 18, no. 3, pp. 545-592, 2014.

[14] A. Bensoussan, K. C. Wong, S. C. P. Yam, and S. P. Yung, “Timeconsistent portfolio selection under short-selling prohibition: from discrete to continuous setting," SIAM Journal on Financial Mathematics, vol. 5, no. 1, pp. 153-190, 2014.

[15] H. Wang and Z. Wu, "Partially observed time-inconsistency recursive optimization problem and application," Journal of Optimization Theory and Applications, vol. 161, no. 2, pp. 664687, 2014.

[16] X. Cui, D. Li, and X. Li, "Mean-variance policy for discretetime cone-constrained markets: time consistency in efficiency and the minimum-variance signed supermartingale measure," Mathematical Finance, 2015.

[17] X. Cui, L. Xu, and Y. Zeng, "Continuous time mean-variance portfolio optimization with piecewise state-dependent risk aversion," Optimization Letters, 2015.

[18] Y. Zeng and Z. Li, "Optimal time-consistent investment and reinsurance policies for mean-variance insurers," Insurance: Mathematics \& Economics, vol. 49, no. 1, pp. 145-154, 2011.

[19] Z. Li, Y. Zeng, and Y. Lai, "Optimal time-consistent investment and reinsurance strategies for insurers under Heston's SV model," Insurance: Mathematics and Economics, vol. 51, no. 1, pp. 191-203, 2012.
[20] Y. Zeng, Z. Li, and Y. Lai, "Time-consistent investment and reinsurance strategies for mean-variance insurers with jumps," Insurance: Mathematics \& Economics, vol. 52, no. 3, pp. 498-507, 2013.

[21] Y. Li and Z. Li, "Optimal time-consistent investment and reinsurance strategies for mean-variance insurers with state dependent risk aversion," Insurance: Mathematics and Economics, vol. 53, no. 1, pp. 86-97, 2013.

[22] H. Zhao, C. Weng, and Y. Zeng, "Time-consistent investmentreinsurance strategies towards joint interests of the insurer and the reinsurer under CEV models," SSRN 2432207, 2014.

[23] H. Zhao, Y. Shen, and Y. Zeng, "Time-consistent investmentreinsurance strategy for mean-variance insurers with a defaultable security," Journal of Mathematical Analysis and Applications, vol. 437, no. 2, pp. 1036-1057, 2016.

[24] Y. Li, H. Qiao, S. Wang, and L. Zhang, "Time-consistent investment strategy under partial information," Insurance: Mathematics and Economics, vol. 65, pp. 187-197, 2015.

[25] D. Li, X. Rong, and H. Zhao, "Time-consistent reinsuranceinvestment strategy for a mean-variance insurer under stochastic interest rate model and inflation risk," Insurance: Mathematics and Economics, vol. 64, pp. 28-44, 2015.

[26] Z. Liang and M. Song, "Time-consistent reinsurance and investment strategies for mean-variance insurer under partial information," Insurance: Mathematics and Economics, vol. 65, pp. 66-76, 2015.

[27] D. Li, X. Rong, and H. Zhao, “Time-consistent reinsuranceinvestment strategy for an insurer and a reinsurer with meanvariance criterion under the CEV model," Journal of Computational and Applied Mathematics, vol. 283, pp. 142-162, 2015.

[28] J. Wei, K. C. Wong, S. C. P. Yam, and S. P. Yung, "Markowitz's mean-variance asset-liability management with regime switching: a time-consistent approach," Insurance: Mathematics and Economics, vol. 53, no. 1, pp. 281-291, 2013.

[29] T. A. Pirvu and H. Zhang, "Utility indifference pricing: a time consistent approach," Applied Mathematical Finance, vol. 20, no. 4, pp. 304-326, 2013.

[30] S. Chen, Z. Li, and Y. Zeng, "Optimal dividend strategies with time-inconsistent preferences," Journal of Economic Dynamics and Control, vol. 46, pp. 150-172, 2014.

[31] D.-M. Dang and P. A. Forsyth, "Continuous time meanvariance optimal portfolio allocation under jump diffusion: an numerical impulse control approach," Numerical Methods for Partial Differential Equations, vol. 30, no. 2, pp. 664-698, 2014.

[32] J. J. Choi, "Diversification, exchange risk and corporate international investment," Journal of International Business Studies, vol. 20, no. 1, pp. 145-155, 1989.

[33] M. Bellalah and Z. Wu, "A simple model of corporate international investment under incomplete information and taxes," Annals of Operations Research, vol. 165, no. 1, pp. 123-143, 2009.

[34] Z. Wu and L. Zhang, "The corporate optimal portfolio and consumption choice problem in the real project with borrowing rate higher than deposit rate," Applied Mathematics and Computation, vol. 175, no. 2, pp. 1596-1608, 2006.

[35] D. Zhang and T. Zhang, "Optimal portfolio of corporate investment and consumption under market closure," International Journal of Business, vol. 17, no. 1, pp. 25-38, 2012.

[36] Z. Huang and D. Zhang, "Optimal portfolio of corporate investment and consumption problem under market closure: inflation case," Mathematical Problems in Engineering, vol. 2013, Article ID 715869, 9 pages, 2013. 
[37] T. Björk, A. Murgoci, and X. Y. Zhou, "Mean-variance portfolio optimization with state-dependent risk aversion," Mathematical Finance, vol. 24, no. 1, pp. 1-24, 2014. 


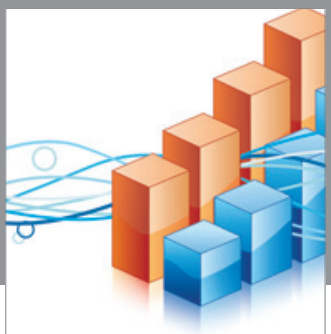

Advances in

Operations Research

vatem alat4

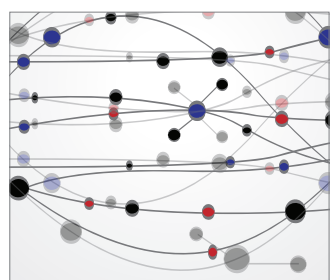

\section{The Scientific} World Journal
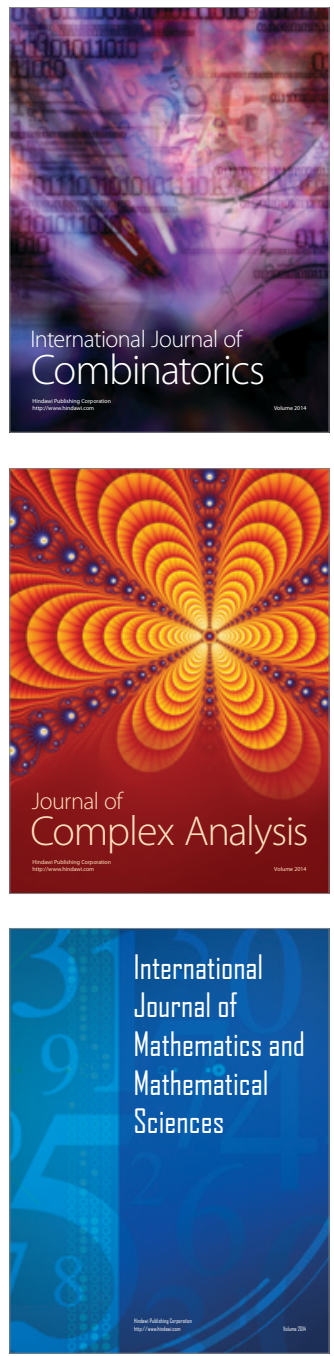
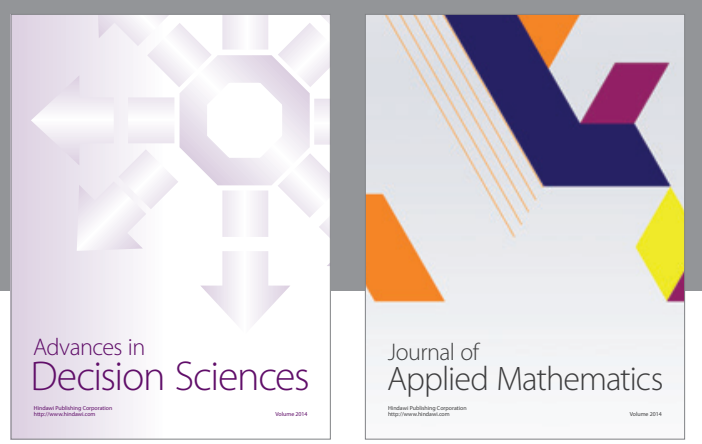

Algebra

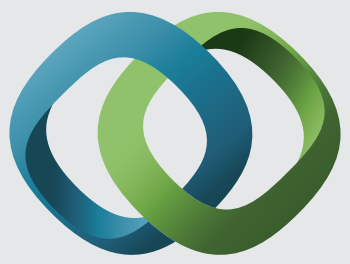

\section{Hindawi}

Submit your manuscripts at

http://www.hindawi.com
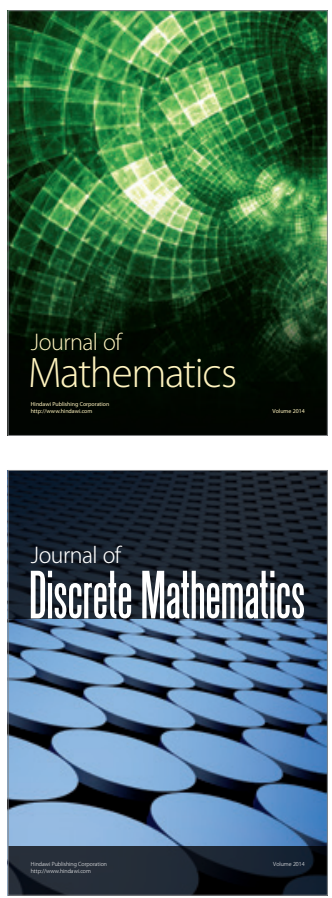

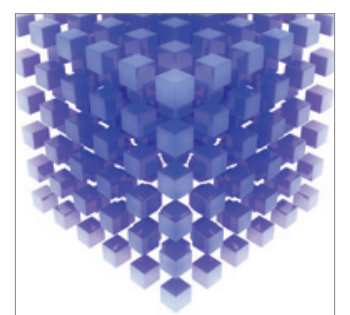

Mathematical Problems in Engineering
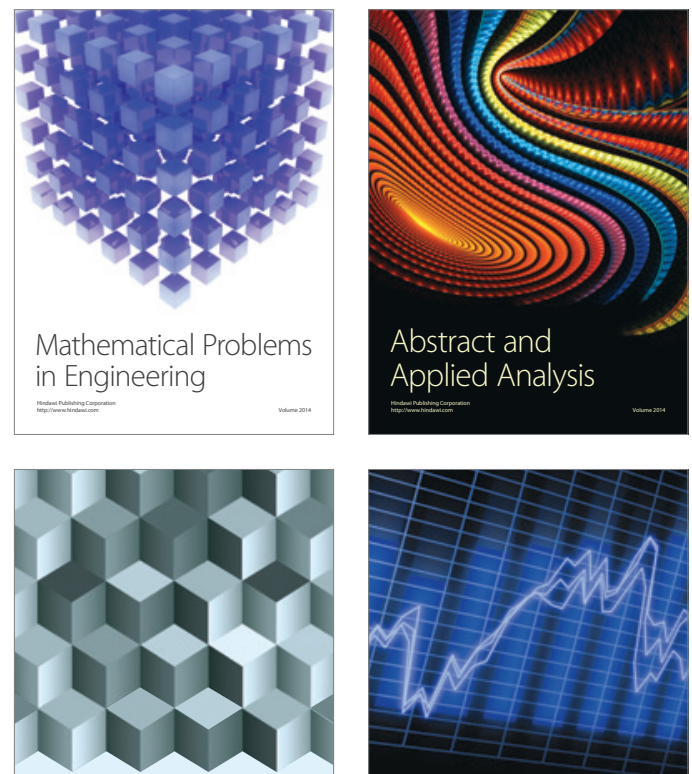

Journal of

Function Spaces

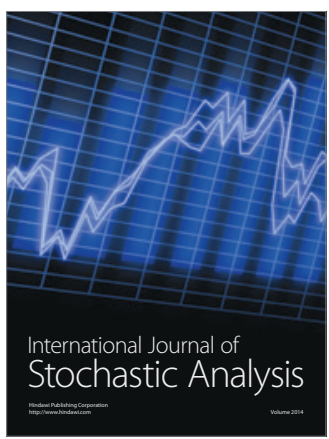

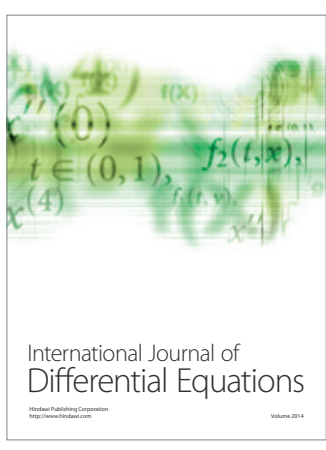
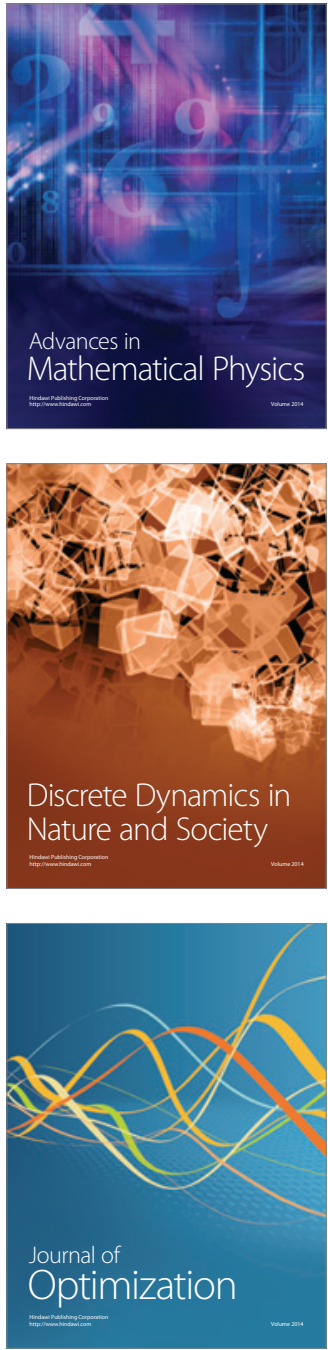